\title{
A systematic RNAi synthetic interaction screen reveals a link between p53 and snoRNP assembly
}

\author{
Dragomir B. Krastev ${ }^{1,2}$, Mikolaj Slabicki ${ }^{2}$, Maciej Paszkowski-Rogacz ${ }^{1,2}$, Nina C. Hubner ${ }^{3,4}$, Magno Junqueira ${ }^{2,4}$, \\ Andrej Shevchenko ${ }^{2}$, Matthias Mann ${ }^{3}$, Karla M. Neugebauer ${ }^{2}$ and Frank Buchholz ${ }^{1,2,5}$
}

TP53(tumour protein 53) is one of the most frequently mutated genes in human cancer and its role during cellular transformation has been studied extensively. However, the homeostatic functions of p53 are less well understood. Here, we explore the molecular dependency network of TP53 through an RNAi-mediated synthetic interaction screen employing two HCT116 isogenic cell lines and a genome-scale endoribonuclease-prepared short interfering RNA library. We identify a variety of TP53 synthetic interactions unmasking the complex connections of p53 to cellular physiology and growth control. Molecular dissection of the TP53 synthetic interaction with UNRIP indicates an enhanced dependency of TP53-negative cells on small nucleolar ribonucleoprotein (snoRNP) assembly. This dependency is mediated by the snoRNP chaperone gene NOLC1 (also known as NOPP140), which we identify as a physiological p53 target gene. This unanticipated function of TP53 in snoRNP assembly highlights the potential of RNAi-mediated synthetic interaction screens to dissect molecular pathways of tumour suppressor genes.

More than 30 years of intense research on the tumour suppressor gene TP53 has revealed its relevance in many aspects of tumour biology (reviewed in ref. 1). It is now well established that p53 (the protein product of TP53) activation leads to the execution of a complex genetic program that limits cellular proliferation and leads to senescence or apoptosis ${ }^{2,3}$. Several lines of evidence have pointed to an under-appreciated homeostasis function of p53 in normal, unstressed cells ${ }^{4}$. As a transcription factor, p53 can mediate the expression levels of its target genes, thereby adjusting cellular homeostasis of basic metabolism ${ }^{5}$, balance of reactive oxygen species $^{6,7}$, stem cell identity and reprogramming ${ }^{8}$ and animal reproduction ${ }^{9}$. These data establish an important role for p53 in cellular homeostasis and indicate that p53-loss-of-function cells become genetically rewired in a way that discriminates them from wild-type cells, a premise that could be explored for the identification of possible p53 genetic dependencies.

Synthetic interaction screens have led the way in identifying new and unanticipated functional interactions between seemingly unrelated protein complexes in yeast ${ }^{10,11}$. In mammalian cells, RNA interference (RNAi)-based synthetic interaction screens are a promising approach to dissect genetic dependencies and the first studies on mammalian synthetic interactions of oncogenes have demonstrated the power of this approach ${ }^{12,13}$. Furthermore, synthetic lethal screens have identified genes that sensitize cells to different chemical drugs ${ }^{14,15}$. Several synthetic interactions with tumour suppressor genes have also been published ${ }^{11,16}$, but to our knowledge a genome-scale survey for synthetic interactions of a tumour suppressor gene has not been reported. Here, we present the results of a systematic investigation of TP53 genetic dependencies and characterize in molecular detail a homeostatic function of p53 in snoRNP biogenesis.

\section{RESULTS}

\section{Genome-scale synthetic interaction RNAi screen with TP53}

We carried out a genome-scale RNAi screen in two HCT116 cell lines that differ only in their TP53 status: TP53 wild type and its TP53-knockout counterpart (ref. 17). The wild-type cells show a robust p53-dependent response and both lines allow efficient transcript depletion through RNAi (Supplementary Fig. S1a-c). An endoribonuclease-prepared short interfering RNA (esiRNA) library was used to interrogate which genes are needed for proliferation of either cell type and thus discriminate the specific genetic requirement of each gene in accordance with the presence or absence of TP53. Two major features were implemented into the screen design. First, the cells were labelled with fluorescent proteins (nuclear monomeric red fluorescent protein, mRFP, for the wild-type and green fluorescent protein, GFP, for the knockout cells), which allowed

${ }^{1}$ University of Technology Dresden, University Hospital and Medical Faculty Carl Gustav Carus, Department of Medical Systems Biology, Fetscherstraße 74, D-01307 Dresden, Germany. ${ }^{2}$ Max Planck Institute of Molecular Cell Biology and Genetics, Pfotenhauerstrasse 108, D-01307 Dresden, Germany. ${ }^{3}$ Max Planck Institute of Biochemistry, Am Klopferspitz 18, 82152 Martinsried, Germany. ${ }^{4}$ Present addresses: Universitair Medisch Centrum Utrecht, 3508 AB Utrecht, The Netherlands (N.C.H.); Brazilian Center for Protein Research, Department of Cell Biology, University of Brasilia, 70910-900 Brasilia, DF, Brazil (M.J.).

${ }^{5}$ Correspondence should be addressed to F.B. (e-mail: buchholz@mpi-cbg.de) 

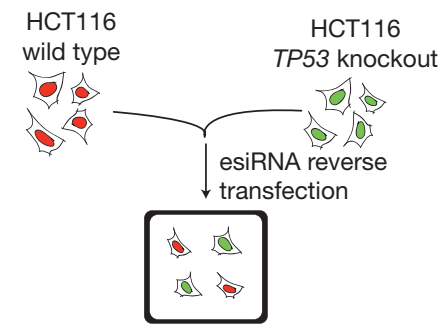

$96 \mathrm{~h}$

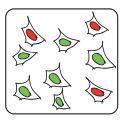

$<-1.3$

Activators

of p53

78 genes

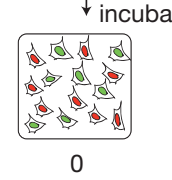

No p53 modulation

15,856 genes

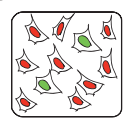

$>0.7 \log _{2}$ (wild type/knockout)

Automated imaging b

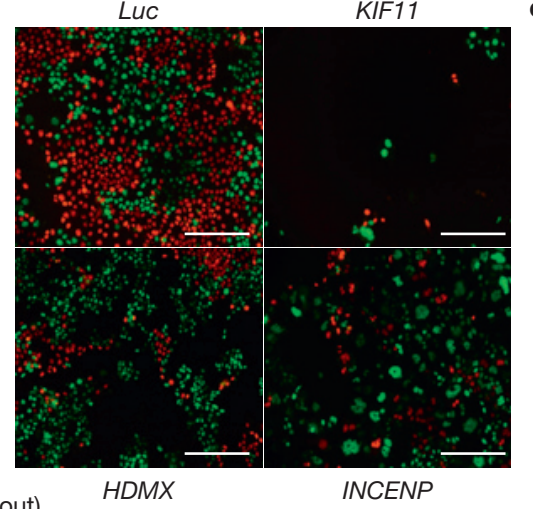
Synthetic sick

with p53

3 genes
Figure 1 Genome-scale TP53 synthetic interaction screen. (a) Schematic representation of the screen assay. Equal numbers of HCT116 wild-type (red) and knockout (green) cells were reverse transfected with esiRNAs. Possible scenarios and the number of knockdowns causing indicated phenotypes are shown. Screen controls are shown in Supplementary Fig. Sla-c. (b) Representative images from transfections with esiRNAs of the non-targeting luciferase control ( $L u c)$, the kinesin family member 11 (KIF11), the p53 regulator

a microscopy-based readout at single-cell resolution. Second, the wild-type and knockout cells were mixed in a 1:1 ratio and transfected with esiRNAs in the same well, ensuring that their viability is compared under precisely the same conditions, reducing the technical variability and significantly improving the identification of genetic interactions (Fig. 1a). The robustness of the assay was first evaluated by treatment with Nutlin-3 $\alpha$ (ref. 18) or HDMX depletion ${ }^{19}$. As expected, both treatments triggered a 533 response and led to significant retardation of proliferation of the wild-type, but not the knockout, cells, thus changing the starting ratio of $1: 1$ to $0.1: 1$ in favour of the knockout cells (Supplementary Fig. S1a-c). Depletion of essential genes, such as kinesin family member 11 (KIF11), equally affected wild-type and knockout cells (Fig. 1b), excluding the possibility that one cell line is more sensitive to the transfection protocol. Thus, the assay is amenable for a high-throughput RNAi screen and the wild-type/knockout ratio is a robust readout of $\mathrm{p} 53$ activation.

The esiRNA library was transfected in duplicate and automated image analysis was used to extract the numbers of wild-type and knockout cells at $96 \mathrm{~h}$ post-transfection ${ }^{20-22}$ (Fig. 1a-c and Supplementary Table S1). A total of 780 (4.8\%) knockdowns affected the cell viability of both cells types, with $276(1.7 \%)$ leading to overall cell counts that prohibited meaningful analyses of altered wild-type/knockout ratios (Supplementary Table S1, viability column). Reactome ${ }^{23}$ and STRING (ref. 24) database analyses revealed that genes encoding core proteins of complexes involved in cell cycle progression, general transcription, messenger RNA processing and protein translation (for example, almost all core subunits of the ribosome, Table 1) were highly enriched in reduced-viability phenotypes in both cell lines. These genes therefore represent the molecular machineries that drive basic cellular proliferation and were excluded from further analysis.

Using an outlier- and asymmetry-insensitive quartile-based technique ${ }^{25}$ to identify primary hits, followed by stringent validation, we identified 81 knockdowns that led to viability phenotypes in
HDMX and the inner centromere protein antigens $135 / 155 \mathrm{kDa}$ ( INCENP) exemplifying knockdown phenotypes (scale bars, $100 \mu \mathrm{m}$ ). (c) Statistical evaluation of the screening results. A histogram of the $\log _{2}$ (wildtype/knockout) calculated in a plate-wise manner is shown. The Gaussian fit to the distribution (red line) underlines the need to use an asymmetry- and outlier-insensitive quartile ( $Q$ )-based threshold (dashed lines, see Methods) with a targeted error rate of 0.05 to determine the primary hits.

only one of the two cell types (Supplementary Table S2). Depletion of 78 genes specifically decreased proliferation and/or viability of the wild-type cells with little to no effect on the knockout cells. These genes represent the genetic interaction network that specifically synergize with TP53 and represent the multitude of pathways that p53 monitors or regulates under normal growth conditions. Interestingly, many of the 78 hit genes that reduced the wild-type/knockout ratio are also involved in basic cellular processes, but not as core components. For example, the depletion of a number of genes encoding ribosome assembly and ribosome maturation factors, such as PES1 (pescadillo homologue 1, containing BRCT domain), RRS1 (RRS1 ribosome biogenesis regulator homologue) and RPF1 (ribosome production factor 1 homologue), decreased the wild-type/knockout ratio (Table 1). These genes are positioned upstream of the mature ribosome subunits that are known to activate the ribosomal protein (RP)-Hdm2-p53 pathway (reviewed in ref. 26), but downstream of the ribosomal RNA transcription machinery. There are more than 170 ribosome assembly factors ${ }^{27}$ and the finding that only a small number of them induce p53 in our screen indicates that p53 monitors specific steps in the assembly process. Interference with their functions propagates signalling through the $\mathrm{p} 53$ pathway, explaining the wild-type-specific phenotype. Therefore, the genes that decreased the wild-type/knockout ratio can be termed activators of p53. They have roles in cellular processes that are guarded by p53 under normal growth conditions and probably act as p53 cell stress sensors in basic cellular processes.

\section{Knockdowns that selectively affect TP53-knockout cells}

The fact that many more knockdowns specifically affected the wild-type cells underscores the crucial role of p53 as a guardian of cell proliferation. However, examination of the screening results also revealed three genes that increased the wild-type/knockout ratio. Knockdown of UNRIP (also known as STRAP, serine/threonine 


\begin{tabular}{|c|c|c|}
\hline & Ribosome (mature ribosome) & Ribosome assembly factors \\
\hline Phenotype & Viability & Decreased wild-type/knockout ratio \\
\hline Wild-type cells & Cell death & Reduced viability \\
\hline Knockout cells & Cell death & Viable \\
\hline Genes & $\begin{array}{l}\text { RPL3, RPL5, RPL6, RPL7, RPL7A, RPL9, RPL11, RPL17, RPL18, } \\
\text { RPL18A, RPL21, RPL23, RPL24, RPL30, RPL31, RPL32, RPL37, } \\
\text { RPL37A, RPSA, RPS3, RPS3A, RPS4X, RPS6, RPS7, RPS8, RPS10, } \\
\text { RPS11, RPS12, RPS15A, RPS16, RPS19, RPS20, RPS26, RPS27A }\end{array}$ & $\begin{array}{l}\text { PES1, RRS1, UTP15, NOP2, RPF1, FBL, } \\
\text { MRM1, RPL24, NSA2, RPL35A, GTPBP4, } \\
\text { EBNA1BP2, ISG20L2, POLR1A, TWISTNB }\end{array}$ \\
\hline Enrichment ( $P$ value) & $1.8 \times 10^{-44}$ & $3.9 \times 10^{-9}$ \\
\hline
\end{tabular}

Gene Ontology term enrichment of the two groups of genes in the viability list and the list of genes decreasing the wild-type/knockout ratio, respectively, is presented by their $P$ values.

kinase receptor associated protein), MASTL (microtubule-associated serine/threonine kinase-like) and KIAA1344 (also known as TXNDC16, thioredoxin domain containing 16) had no effect on the growth of the wild-type cells, whereas expression of these genes was required for efficient growth of the knockout cells (Fig. 2a), raising the possibility that they act in conjunction with TP53 to maintain cellular fitness. Indeed, time-lapse video microscopy revealed that for all three knockdowns the proliferation rate of the knockout cells was reduced, whereas the wild-type cells grew with similar proliferation rates as the control transfected cells (Fig. 2b). TUNEL (TdT-mediated dUTP nick end labelling) staining showed no significant increase in the level of apoptosis, further supporting that the phenotypes are caused by slower growth of the knockout cells (Supplementary Fig. S2a).

\section{UNRIP influences the localization of the SMN complex}

The knockdown of UNRIP was selected for further analysis because it gave the strongest phenotype and it has not been linked to p53 thus far. Two independent and non-overlapping esiRNAs efficiently depleted both UNRIP transcript and protein levels (Fig. 2c,d and Supplementary Fig. S2d,e). Detailed analysis of the time-lapse videos showed that the doubling time of knockout cells was extended by about $30 \%$ (36 versus $28 \mathrm{~h}$ in UNRIP versus luciferase knockdown), thus resulting in a decrease in cell number to about $70 \%$ in comparison with the wild-type cells and control-transfected knockout cells (Fig. 2b). Neither a particular cell cycle arrest, nor an increase in the number of apoptotic cells, was observed during the time-lapse analysis and on TUNEL/propidium iodide staining (Supplementary Fig. S2a,b). Furthermore, no induction of p $21^{\text {WAF1 }}$ (also known as cyclin-dependent kinase inhibitor 1) was observed in UNRIP-depleted knockout cells (Supplementary Fig. S2f-h).

To substantiate the synthetic effect of TP53 and UNRIP, we carried out clonogenic survival and colony formation assays in isogenic cell lines. The phenotype observed in the competitive growth assay was also seen in long-term growth experiments, demonstrating that the effect can be replicated with different assays (Fig. 2e). Consistent results were seen with two independent RNAi triggers (short hairpin RNAs and esiRNA) and in an independent pair of isogenic cell lines differing solely in their TP53 status (RKO cells) ${ }^{28}$, signifying that the TP53-UNRIP synthetic interaction is reproducible and not HCT116 specific. Furthermore, TP53 and UNRIP co-depletion in HCT116 wild-type cells resulted in decreased clonogenic survival, showing that their genetic interaction is not a result of a long-term adaptation to in vitro culturing conditions, but rather a direct genetic interaction (Supplementary Fig. S2c). Taken together, these experiments support a cooperative role of TP53 and UNRIP in the maintenance of cellular fitness and proliferation.

UNRIP has been shown to act as a negative regulator of TGF $\beta$ (transforming growth factor $\beta$ ) signalling ${ }^{29}$. However, none of the employed assays revealed a significant change in TGF $\beta$ signalling on UNRIP depletion (Supplementary Fig. S2h-j).

UNRIP has also been associated with the survival of motor neurons (SMN) complex in HeLa cells ${ }^{30}$. To investigate a potential association of UNRIP with SMN in HCT116 cells, we generated localization and affinity purification (LAP)-tagged UNRIP (UNRIP-LAP) cell lines using the TransgeneOmics approach ${ }^{31,32}$. Immunoprecipitation, followed by protein interaction analyses using mass spectrometry, revealed that human as well as mouse UNRIP-LAP efficiently co-purified the complete SMN complex (SMN1; SIP1, survival of motor neuron protein interacting protein 1, also known as GEMIN2; DDX20, DEAD (Asp-Glu-Ala-Asp) box polypeptide 20, also known as GEMIN3; and gem (nuclear organelle)-associated proteins 4-8, GEMIN4-8) and many of its substrates (Supplementary Table S3). Thus, UNRIP physically interacts with SMN components and its substrates in HCT116 cells.

The SMN complex functions as an assembly chaperone mainly for the small nuclear RNPs (snRNP; ref. 33). Most SMN complex subunits, including SMN1, show a prominent accumulation in the Cajal body ${ }^{34}$. Indeed, SMN1 antibody staining revealed 3-4 Cajal bodies in wild-type and knockout cells, and UNRIP depletion did not alter this distribution in wild-type cells (Fig. 3a). In striking contrast, UNRIP depletion in knockout cells led to a significant reduction in the number of SMN1-positive Cajal bodies, with roughly one-third of the cells showing no nuclear SMN1-positive staining (Fig. 3b). Virtually identical results were obtained on examination of the Gemin2 component of the SMN complex, but not the bona fide structural component of Cajal bodies-P80/COIL (Supplementary Fig. S3a,b). Therefore, the whole SMN complex is specifically affected on UNRIP depletion in knockout cells without affecting the overall Cajal body structure. Similar results were obtained with RKO cells, in which UNRIP depletion led to a significant decrease in the amount of nuclear SMN1 specifically in TP53-knockout cells (Supplementary Fig. S3c). Cross-species RNAi rescue experiments ${ }^{31}$ excluded possible RNAi offtarget effects as a reason for this observation (Supplementary Fig. S3d). Finally, co-depletion of TP53 and UNRIP led to a significant reduction of SMN1-positive Cajal bodies in the wild-type cells, demonstrating that the observed phenotype is a primary effect of the lack of both p53 and UNRIP (Fig. 3a,b). Hence, simultaneous loss of p53 and UNRIP reduces the number of $\mathrm{SMN}$-complex-positive Cajal bodies. 
a

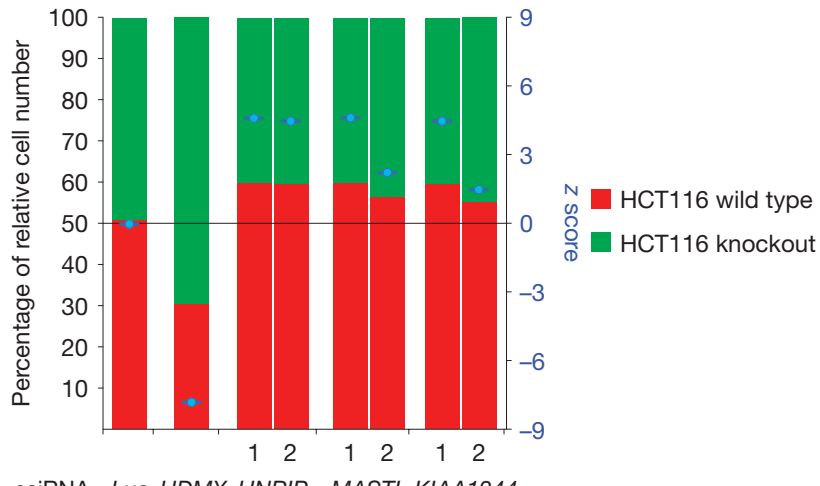

EsiRNA LUC HDMX UNRIP MASTL KIAA1344

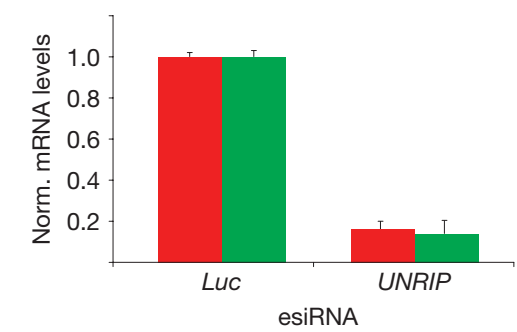

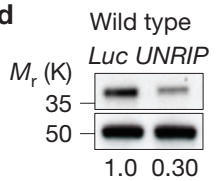

Knockdown HCT116

$M_{r}(\mathrm{~K})$ Luc UNRIP esiRNA

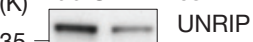

$50-$ Tubulin

$1.0 \quad 0.31$ knockdown

b
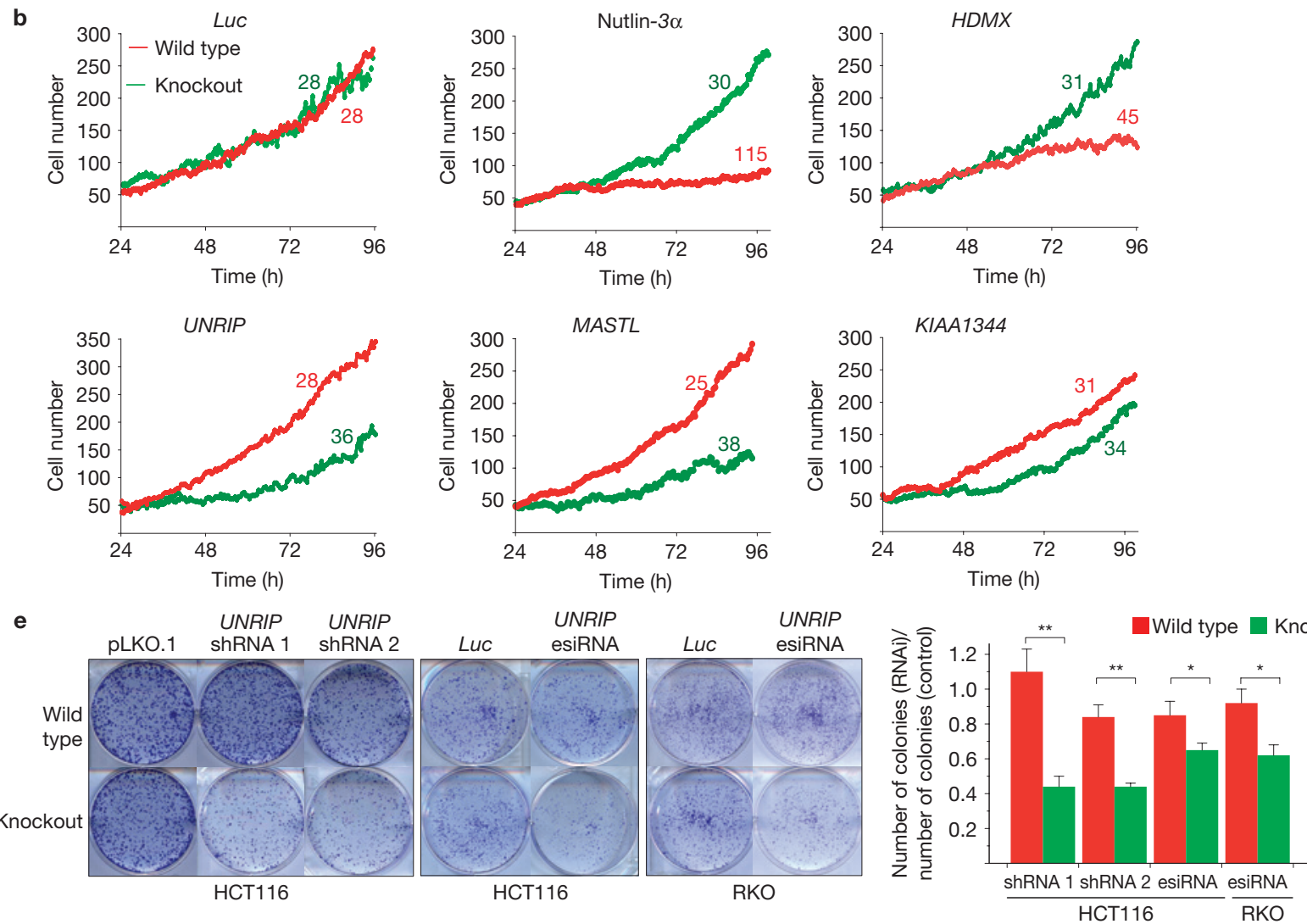

Figure 2 Depletion phenotypes of knockdowns increasing the wild-type/knockout ratio. (a) RNAi phenotype of the three genes decreasing the number of knockout cells-UNRIP, MASTL and KIAA1344. The cell number for each cell type is presented as a percentage of the total cells counted per well (left $y$ axis) and the corresponding $z$ score (right $y$ axis, calculated versus LuC knockdown, see Methods). The knockdown of HDMX is shown as a positive control. Two esiRNAs (1 and 2) are shown for each hit gene. (b) Time-lapse analysis of the RNAi phenotypes. Wild-type and knockout cells transfected with the indicated esiRNAs were imaged for a period of $72 \mathrm{~h}$, starting at $24 \mathrm{~h}$ post-transfection. The doubling time was calculated from an exponential fit of the growth

\section{UNRIP influences SMN complex shuttling in a p53-dependent manner}

Assembly of the Sm-ring onto the small nuclear RNA (snRNA) and the hypermethylation of the cap to trimethylguanosine (TMG) are two prerequisites for nuclear import of the newly assembled curves (values next to the curves). (c,d) Quantification of UNRIP knockdown. mRNA and protein depletion levels normalized to luciferase-depleted cells. GAPDH or $\alpha$-tubulin served as an internal loading control (mean \pm s.d., $n=3$ ). (e) Long-term survival (colony formation assay) of HCT116 and RKO cells on UNRIP depletion. The quantification presents the number of colonies relative to control transfection. The significance is determined with Student's two-tailed $t$-test, * $P$ values $<0.05$, ** $P$ values $<0.01$ (mean \pm s.d., $n=3$ ). The depletion phenotypes are further characterized in Supplementary Fig. S2. In all panels, red and green represent wild-type and knockout cells, respectively. Uncropped images of blots are shown in Supplementary Fig. S7a.

snRNP particles to the Cajal body $y^{35-37}$. To determine whether UNRIP depletion blocks cytoplasmic steps of snRNP assembly, we measured the snRNP assembly rates in metabolically labelled wild-type and knockout cells on UNRIP depletion. Anti-Sm (Y12) and anti-TMG antibodies recovered equal amounts of snRNA from 

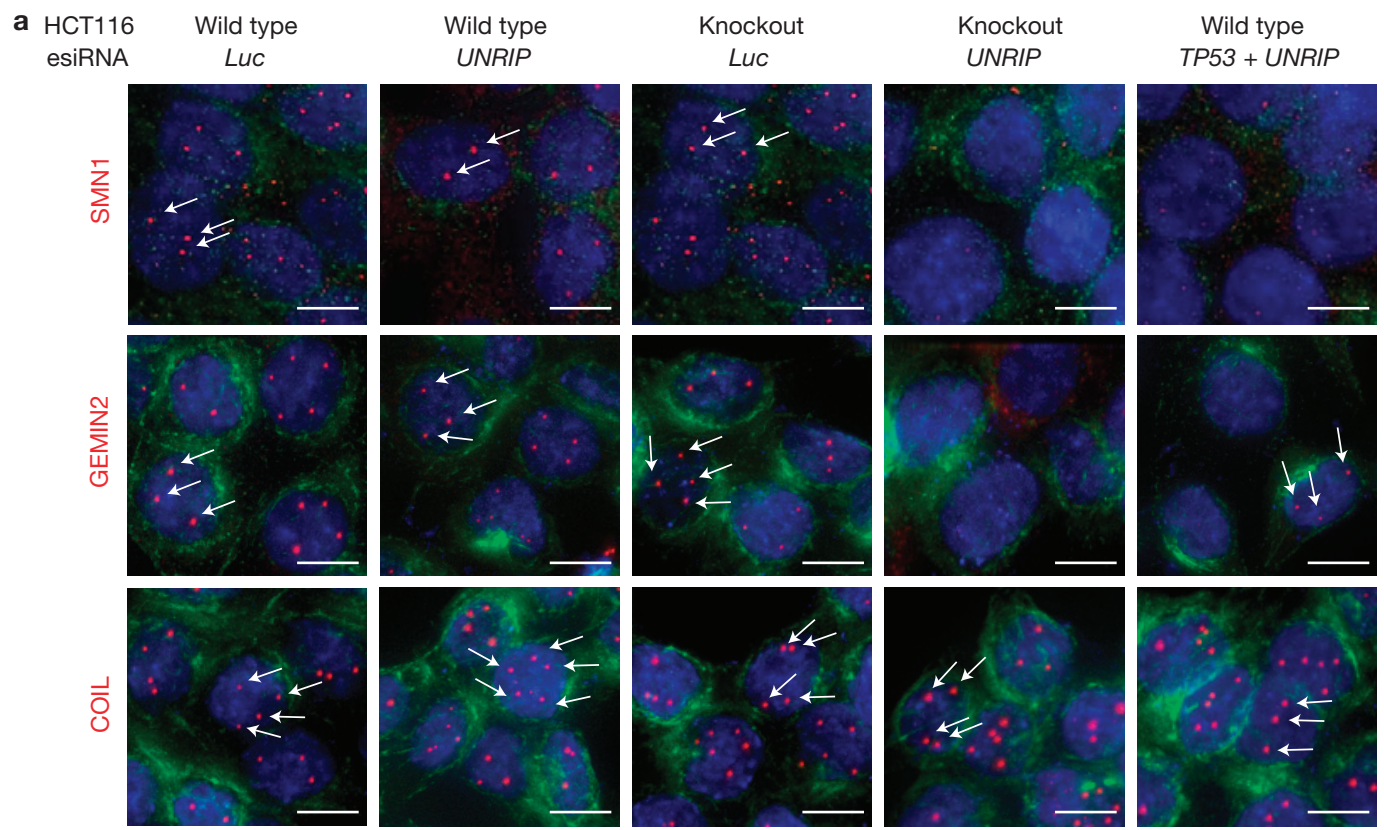

b
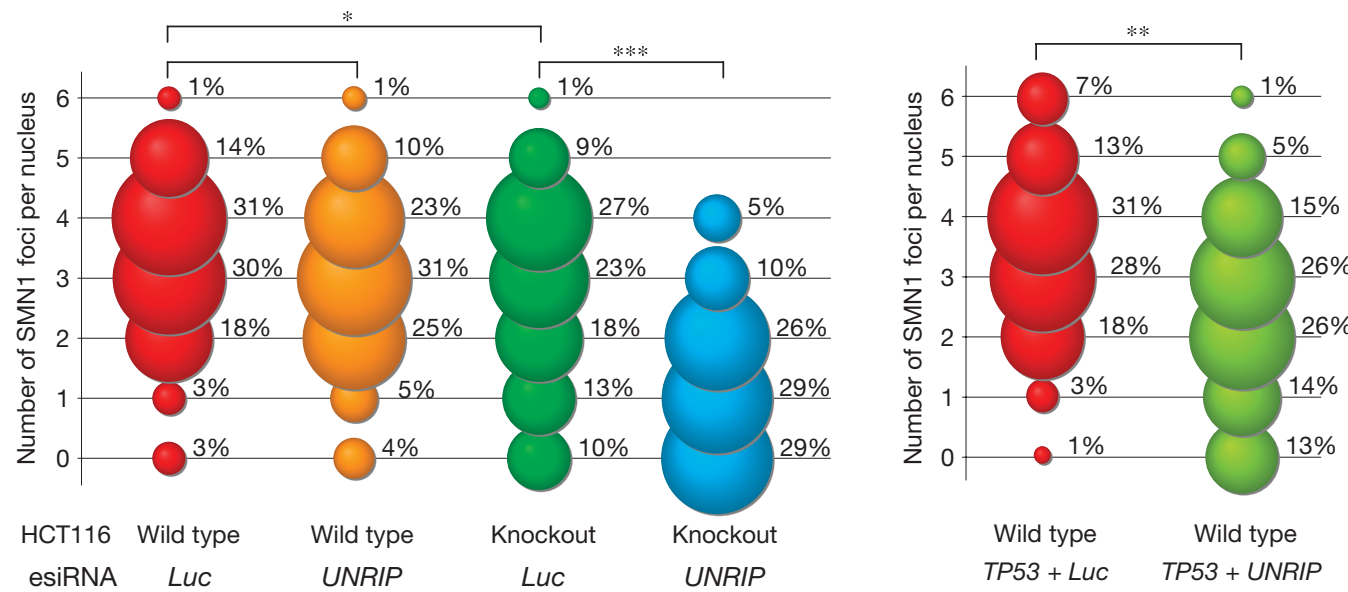

C

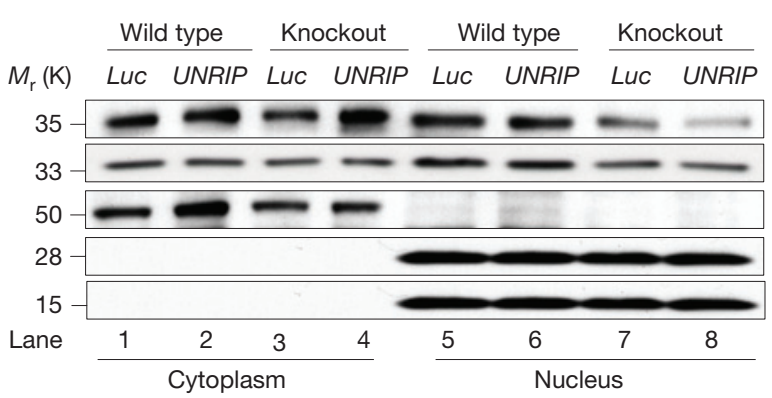

Figure 3 SMN complex localization on UNRIP depletion.

(a) Immunofluorescence microscopy analysis of the cellular localization of SMN1, Gemin2 and COIL in wild-type and knockout cells on UNRIP depletion. Cells were stained with DAPI (4,6-diamidino-2-phenylindole; blue), $\alpha$-tubulin antibody (green) and antibodies against the indicated proteins (red) for illustrated cells and knockdowns. Note that SMN1 and Gemin2 Cajal body (white arrows) localization is affected by UNRIP depletion only in knockout cells. Scale bars, $5 \mu \mathrm{m}$. (b) Quantification of the number of SMN1-positive Cajal bodies per nucleus. The ball size corresponds to the percentage of cells with a particular number of foci (numerically shown on the right of each ball). The distributions of indicated cells and knockdowns were compared with Student's two-tailed
HCT116 esiRNA

SMN1

PCNA

Tubulin

$\mathrm{SmB}$

Histone 3

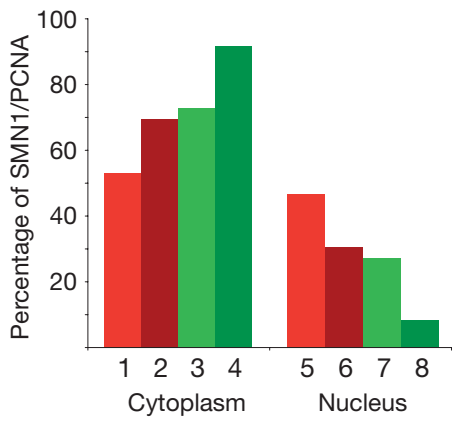

$t$-test, ${ }^{*} P$ values $<0.01, * * P$ values $<10^{-5}, * * * P$ values $<10^{-10}$, 200-300 nuclei counted per condition in three independent experiments. Quantification of Gemin2 and COIL foci is provided in Supplementary Fig. S3a,b. (c) SMN1 nucleo-cytoplasmic distribution on UNRIP depletion. Western blot analyses of indicated proteins in cytoplasmic and nuclear extracts of control ( $L u c$ ) and UNRIP-depleted wild-type and knockout cells are shown. Probing with anti-tubulin, anti-histone 3 and anti-SmB (small nuclear ribonucleoprotein polypeptides B and B1) revealed the purity of the fractions. The right panel represents quantification of SMN1 in each fraction, normalized to the amount of proliferating cell nuclear antigen (PCNA) as the loading control. Uncropped images of blots are shown in Supplementary Fig. S7b. 
a

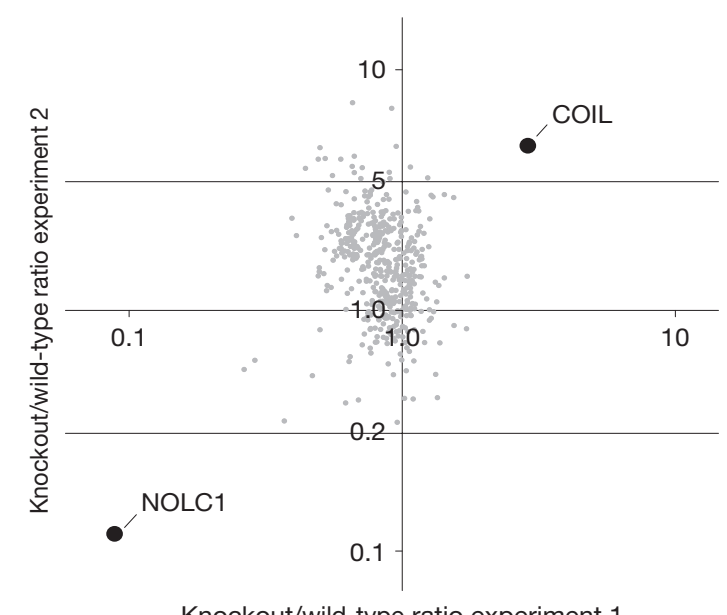

Knockout/wild-type ratio experiment 1

e
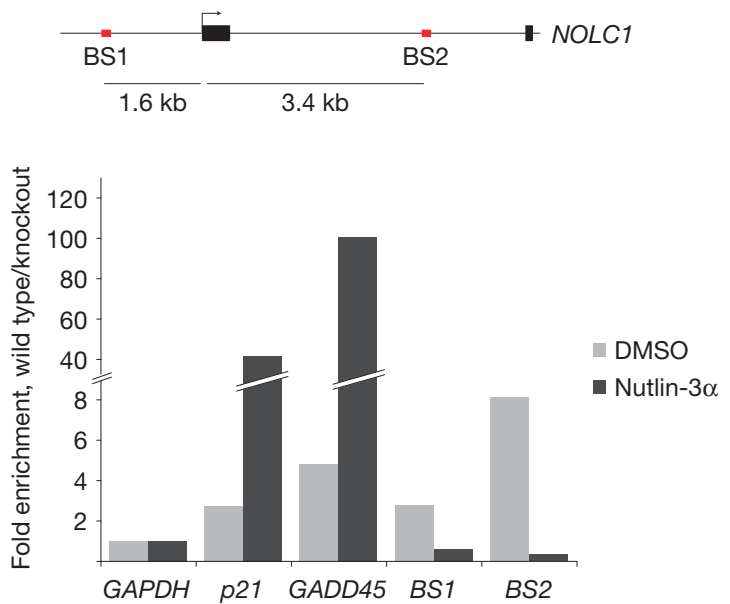

Figure 4 NOLC1 differentially associates with UNRIP in wild-type and knockout cells and NOLC1 is a p53 target gene. (a) Comparative quantitative mass spectrometry of UNRIP protein complexes purified from wild-type and knockout cells. A scatter plot of the correlation of two independent pulldown experiments showing the heavy/light ratio of each identified protein purified from each cell type is illustrated. Immunoprecipitated proteins significantly over-presented (COIL) or under-presented (NOLC1) in the knockout versus the wild-type are highlighted (black dots). (b) Expression analysis of COIL and NOLC1 mRNA. qRT-PCR results for COIL and NOLC1 mRNA levels in HCT116 and RKO wild-type and knockout cells are shown. The values are normalized to the expression levels in the wild-type cells (mean \pm s.d., $n=3$ ). (c) Western blot analysis of COIL, NOLC1 and UNRIP-LAP in HCT116 and RKO wild-type and knockout cells. (d) Immunofluorescence microscopy analysis

all samples, showing that SMN-mediated assembly and maturation of snRNPs occurs independently of UNRIP and p53 (Supplementary Fig. S3e). After snRNP core assembly and TMG cap formation, the snRNP-SMN complex is imported into the nucleus ${ }^{37}$. To determine whether UNRIP plays a role in snRNP-SMN complex nuclear-cytoplasmic transport, we measured the amount of SMN1 in nuclear and cytoplasmic fractions of UNRIP-depleted cells. No prominent differences of nuclear versus cytoplasmic SMN1 were detected in control- and UNRIP-depleted wild-type cells (Fig. 3c, lane 5 and 6). In contrast, the depletion of UNRIP in knockout

f b
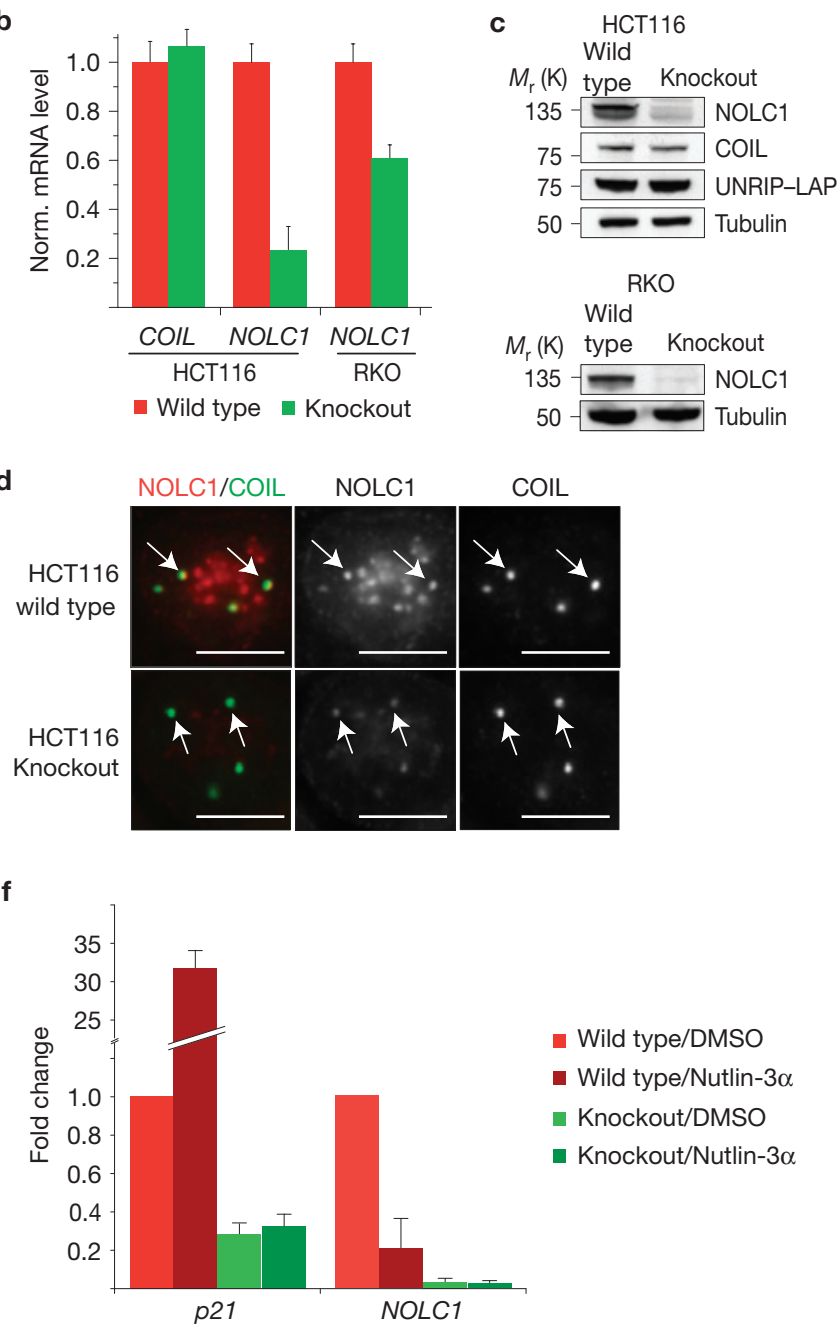

of NOLC1 (red) and COIL (green) in wild-type and knockout cells. The Cajal bodies are indicated with white arrows. Scale bars, $5 \mu \mathrm{m}$. (e) p53 ChIP. Wild-type and knockout cells were treated with DMSO or $5 \mu \mathrm{M}$ Nutlin-3 $\alpha$ for $4 \mathrm{~h}$, p53 was immunoprecipitated (DO-1 antibody) and the enrichment of selected promoter sequences was evaluated using quantitative PCR. The fold enrichment is calculated as a ratio of the percentage inputs from wild-type versus knockout samples. GAPDH was used to normalize this ratio. Shown is a representative experiment $(n=3)$. (f) NOLC1 mRNA transcript levels are p53-dependent. HCT116 cells were treated with $5 \mu \mathrm{M}$ Nutlin-3 $\alpha$ for $4 \mathrm{~h}$ and the expression of p21/WAF1 and NOLC1 mRNA was analysed by qRT-PCR. The values are normalized to DMSO-treated wild-type cells (mean \pm s.d., $n=3$ ). The response to DNA-damaging agents is presented in Supplementary Fig. S4c. Uncropped images of blots are shown in Supplementary Fig. S7c.

cells increased cytoplasmic (Fig. 3c, lane 4 versus 3) and decreased nuclear SMN1 levels (Fig. 3c, lane 8 versus 7), indicating that UNRIP mediates SMN nuclear import or Cajal body retention with the aid of a p53-dependent factor.

\section{NOLC1 mediates the synthetic interaction between UNRIP and TP53}

In the search for a factor that mediates the SMN accumulation in Cajal bodies in a p53-dependent manner, we compared the UNRIP protein interactome in wild-type and knockout cells using 
a

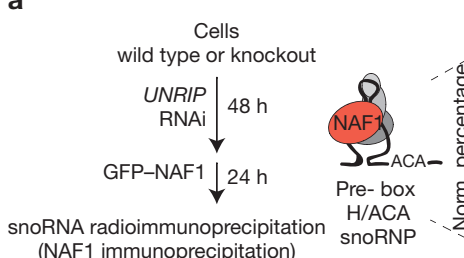
(NAF1 immunoprecipitation)

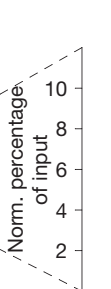

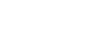

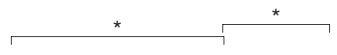

,

ACA21

$\checkmark$ ACA26

- $\quad$ ACA35 $-2-1$

$$
\frac{\text { Luc UNRIP }}{\text { TP53 wild type }} \frac{\text { Luc UNRIP }}{\text { TP53 knockout }}
$$

b
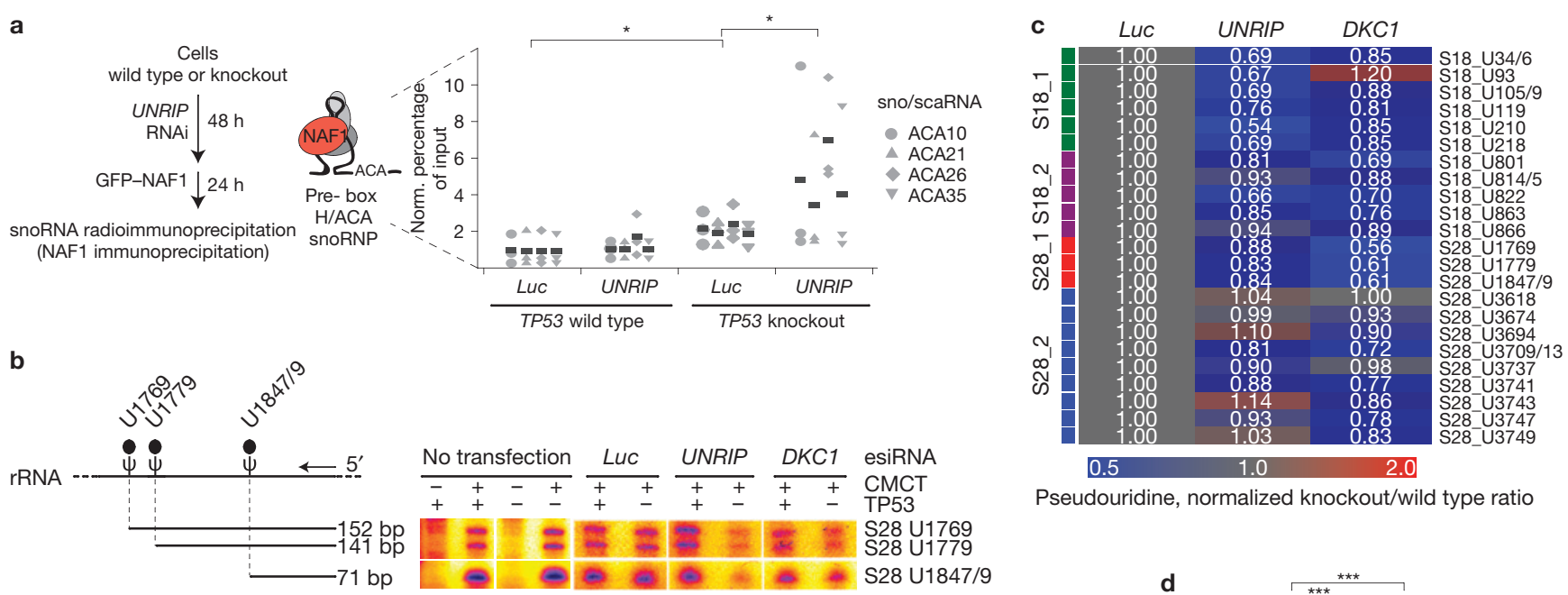

Pseudouridine, normalized knockout/wild type ratio
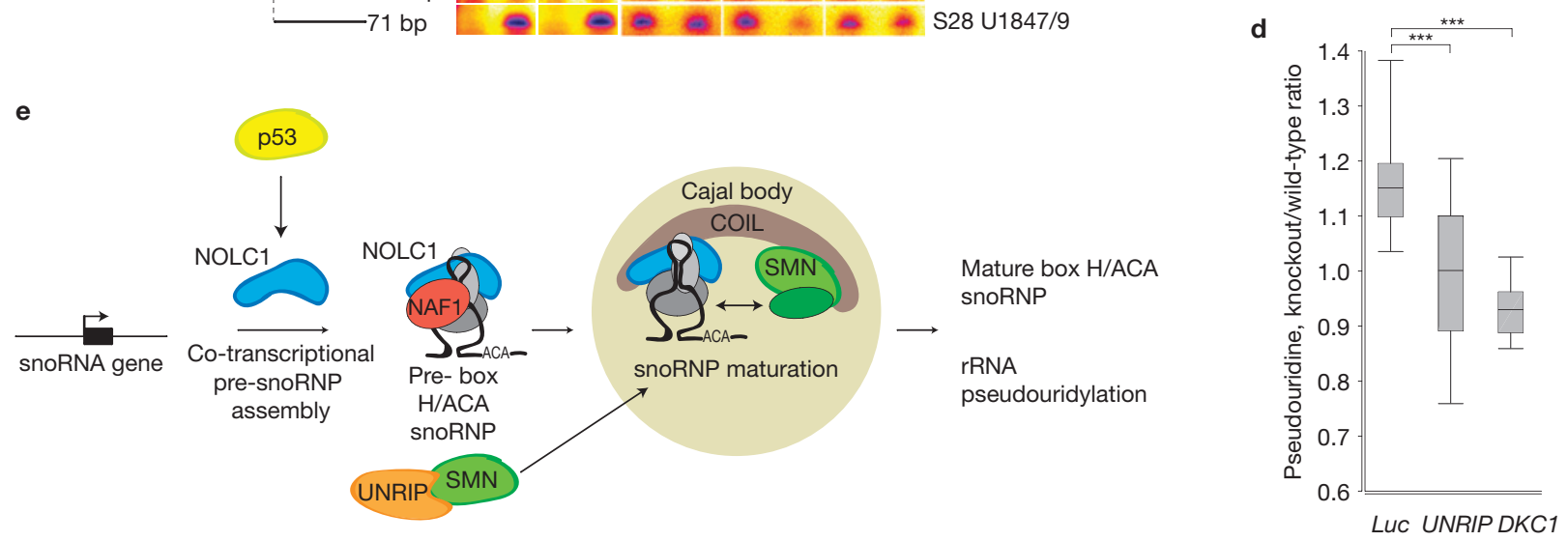

Figure 5 rRNA pseudouridylation is dependent on p53 and UNRIP. (a) snoRNP assembly assay. UNRIP-depleted HCT116 wild-type and knockout cells were transfected with a plasmid expressing GFP-NAF1. After $24 \mathrm{~h}, \mathrm{NAF} 1$ was immunoprecipitated and the amount of associated pre-snoRNAs was investigated by qRT-PCR. The black bars represent mean enrichment values; significance was tested with Student's two-tailed $t$-test, ${ }^{*} P<0.05$, three independent experiments. (b) CMCT assay for pseudouridine $(\psi)$ detection. After CMCT modification of total cellular RNA, primers were hybridized to targeted regions of the rRNA and reverse-transcriptase reactions determined the modified pseudouridine sites (black ball). Equal amounts of RNA were loaded in all reactions. The greyscale intensities are colour-coded for indicated treatments. (c,d) Quantification of the amount of pseudouridylation at 24 sites in $18 \mathrm{~S}$ and $28 \mathrm{~S}$ rRNA on a per site (c) or per treatment (d) basis. The colour-coding represents the knockout/wild-type ratio (blue, decreased;

quantitative BAC (bacterial artificial chromosome) interactomics ${ }^{38}$ (QUBIC). Wild-type and knockout cells expressing LAP-tagged UNRIP were SILAC (stable isotope labelling of amino acids in cell culture)-labelled with either light $\left({ }^{12} \mathrm{C}^{14} \mathrm{~N}\right)$ or heavy $\left({ }^{13} \mathrm{C}^{15} \mathrm{~N}\right)$ isotopes in cell culture, respectively ${ }^{39}$. Anti-GFP pulldowns of 'light' wild-type and 'heavy' knockout cells were carried out. Each peptide was therefore present in a 'light' and 'heavy' form, representing relative protein abundance in wild-type and TP53-knockout cells. Most proteins had a heavy/light ratio of 1:1, as they either show no differential binding in knockout versus wild-type cells or represent background binding to the affinity matrix. For example, UNRIP-LAP interacted with the SMN complex, as well as with other Cajal body proteins, with equal affinity in both cell lines (Fig. 4a and Table 2). Strikingly, two proteins showed significantly different ratios-COIL (ratio of red, increased) of the intensities of the pseudouridine sites for indicated treatments, with the ratio of the Luc control for each site set to 1 . The sites are clustered according to the primer with which they were detected (18S_1, green; 18S_2, purple; 28S_1, red; 28S_2, blue). The box plot shows the averaged values of knockout/wild-type pseudouridine ratios of the 24 tested sites for the indicated treatments. The whiskers denote the 10 th and 90th percentiles of the values, respectively. The significance was tested with Student's paired two-tailed $t$-test, ${ }^{* * *} P<0.001, n=24$, three independent experiments. (e) Model summarizing the synthetic interaction between TP53 and UNRIP. UNRIP is required to bring the SMN complex into the Cajal bodies. TP53 regulates the levels of NOLC1, which brings the immature snoRNPs into the Cajal bodies, where on interaction with COIL and SMN the mature snoRNPs are assembled. Thus, absence of TP53 synergizes with decreased UNRIP levels to perturb snoRNP assembly and in turn this decreases the level of pseudouridylation of rRNA.

about 4) was enriched and nucleolar protein NOLC1 (nucleolar and coiled-body phosphoprotein 1 ; ratio of about 0.1 ) was under-presented in pulldowns from the knockout when compared with wild-type cells. Quantitative PCR with reverse transcription (qRT-PCR), western blot and immunofluorescence microscopy analyses showed that NOLC1, but not COIL, mRNA and protein levels are generally lower in knockout cells in comparison with wild-type cells (Fig. $4 \mathrm{~b}-\mathrm{d}$ ), indicating that $\mathrm{NOLC1}$ is a p53-regulated gene. Importantly, the same reduced NOLC1 levels were also observed in TP53-knockout RKO cells, indicating that this effect is not specific to HCT116 cells (Fig. 4b,c). p53scan software ${ }^{40}$ predicted four putative p53-binding sites within the NOLC1 genomic sequence (Supplementary Fig. S4a,b). Carrying out p53 chromatin immunoprecipitation (ChIP) experiments, we identified significant enrichment for two sites, one located $1.6 \mathrm{~kb}$ upstream 
Table 2 Comparative UNRIP proteomics.

\begin{tabular}{|c|c|c|c|c|c|c|}
\hline \multirow[b]{2}{*}{ Protein } & \multirow[b]{2}{*}{ IPI } & \multirow[b]{2}{*}{ Seq. cov. (\%) } & \multicolumn{2}{|c|}{ ‘Heavy' knockout versus 'light' wild type } & \multicolumn{2}{|c|}{ 'Heavy' wild type versus 'light' knockout (reciprocal values) } \\
\hline & & & Unique peptides & SILAC ratio & Unique peptides & SILAC ratio \\
\hline UNRIP & IPI00294536 & 97 & 29 & 1.000 & 33 & 1.000 \\
\hline UNR & IPI00470891 & 69 & 46 & 1.280 & 52 & 0.802 \\
\hline SMN1 & IPI00003394 & 34 & 11 & 1.094 & 9 & 1.241 \\
\hline GEMIN2 & IPI00024281 & 72 & 14 & 0.985 & 12 & 1.618 \\
\hline GEMIN3 & IPI00005904 & 58 & 35 & 1.214 & 35 & 1.241 \\
\hline GEMIN4 & IPI00027717 & 47 & 39 & 1.163 & 38 & 1.512 \\
\hline GEMIN5 & IPI00291783 & 38 & 14 & 0.977 & 16 & 1.000 \\
\hline GEMIN6 & IPI00103087 & 55 & 8 & 1.203 & 8 & 1.812 \\
\hline GEMIN7 & IPI00003027 & 84 & 6 & 1.185 & 4 & 1.376 \\
\hline GEMIN8 & IPI00301879 & 39 & 4 & 1.130 & 7 & 1.773 \\
\hline SNRPB & IPI00329512 & 39 & 4 & 1.144 & 6 & 1.736 \\
\hline SNRPC & IPI00641788 & 39 & 1 & NA & 2 & 1.618 \\
\hline SNRPD1 & IPI00903286 & 44 & 4 & 1.060 & 3 & 1.736 \\
\hline SNRPD2 & IPI00017963 & 59 & 10 & 1.115 & 10 & 1.232 \\
\hline SNRPD3 & IPI00879750 & 38 & 4 & 1.080 & 5 & 1.486 \\
\hline SNRPE & IPI00029266 & 70 & 3 & 0.989 & 4 & 1.792 \\
\hline SNRPF & IPI00220528 & 58 & 5 & 1.055 & 6 & 2.141 \\
\hline SNRPG & IPI00745343 & 37 & 2 & 1.121 & 4 & 1.603 \\
\hline SNRP70 & IPI00290204 & 75 & 7 & 1.026 & 6 & 1.205 \\
\hline NOLC1 & IPI00216654 & 30 & 12 & 0.086 & 8 & 0.116 \\
\hline COIL & IPI00006442 & 12 & 3 & 2.959 & 4 & 4.854 \\
\hline C1ORF144 & IPI00470594 & 52 & 7 & 0.931 & 6 & 1.309 \\
\hline
\end{tabular}

IPI, International Protein Index; Seq. cov., sequence coverage; NA, not available.

(BS1) and another located $3.4 \mathrm{~kb}$ downstream (BS2) of the NOLC1 transcription initiation start site, respectively (Fig. 4e). Typically, on stress activation, p53 binds to its response elements in target genes and leads to their upregulation or downregulation. Surprisingly, p53 ChIP signals at NOLC1 BS1 and BS2 decreased markedly on p53 induction (Fig. 4e), indicating that p53 leaves these sites under stress conditions. Furthermore, NOLC1 mRNA levels decreased fourfold in p53-stimulated wild-type cells (Fig. 4f and Supplementary Fig. S4c). Consistent with the idea that $\mathrm{p} 53$ vacates the promoter under stress conditions, we observed downregulation of reporter constructs harbouring the BS1 sequence on p53 overexpression (Supplementary Fig. S4d). Collectively, these experiments identify NOLC1 as a p53 target gene whose continuous expression is dependent on physiological levels of p53. To our knowledge, this is the first report of a p53 target gene that is downregulated not by $\mathrm{p} 53$ recruitment and active repression, but rather by $\mathrm{p} 53$ promoter vacation.

\section{snoRNP functions are affected in a p53- and UNRIP-dependent} manner

NOLC1 is a nuclear phosphoprotein that has previously been localized to Cajal bodies and nucleoli ${ }^{41,42}$. Cajal body localization is mediated by direct interactions with COIL and is SMN-dependent ${ }^{43,44}$. As both NOLC1 and SMN interact with core snoRNP components, they may have a role in snoRNP assembly in Cajal bodies ${ }^{45-47}$. NOLC1 has also been suggested to participate in snoRNP trafficking ${ }^{48}$ and stability ${ }^{46}$. We investigated whether any of these functions is the basis for the observed knockout-specific phenotype. The steady-state levels of the mature snoRNAs were not affected by either NOLC1 or UNRIP depletion (Supplementary Fig. S5a). Furthermore, RNA fluorescent in situ hybridization revealed that the cellular localization of five examined snoRNAs was not altered under the same conditions (Supplementary Fig. S5b). Next, we examined possible defects in the assembly of de novo synthesized snoRNPs. To asses the rate of snoRNP assembly, we first measured the association of newly synthesized snoRNAs with NAF1 (nuclear assembly factor 1 homologue), a protein that binds the immature snoRNPs (ref. 49). We transfected control- (Luc) and UNRIP-depleted wild-type or knockout cells with a plasmid expressing GFP-NAF1, followed by NAF1 immunoprecipitation and quantification of associated snoRNAs. Control-depleted knockout cells showed about twofold enrichment of snoRNAs in comparison with control-depleted wild-type cells, indicating a delay in snoRNA maturation, possibly due to the decreased NOLC1 levels (Fig. 5a). This enrichment was further enhanced specifically in UNRIP-depleted knockout cells, indicating that both NOLC1 and UNRIP are needed for efficient snoRNP assembly. Finally, we assessed snoRNA assembly by measuring the amounts of snoRNP-catalysed modifications in the rRNA. Pulse-chase metabolic labelling experiments revealed that rRNA cleavage patterns and rRNA methylation (catalysed by the box C/D snoRNPs) were unaffected in cells of all investigated conditions (Supplementary Fig. S6a-c). Last, we measured the amounts of pseudouridines in functionally important regions of the rRNA (ref. 50), the formation of which is catalysed by the box H/ACA snoRNPs. Figure $5 \mathrm{~b}-\mathrm{d}$ shows the quantified ratio of pseudouridine (knockout/wild-type signal) in UNRIP- and control-depleted cells for 24 sites of $18 \mathrm{~S}$ and $28 \mathrm{~S}$ rRNAs. Remarkably, UNRIP depletion led to decreased levels of pseudouridylation at most of the 24 sites assayed; the effects of UNRIP depletion were often as strong or stronger than the depletion of the enzyme dyskerin (DKC1), which catalyses the reaction ${ }^{51}$. This survey of 24 out of about 100 pseudouridylation sites on rRNA establishes the generality of the effects seen here. The fact that both UNRIP and DKC1 depletion led to decreased levels of pseudouridylation preferentially in the knockout cells, together with the increased association of pre-snoRNAs with NAF1, indicates that snoRNP assembly and subsequent function is the underlying vulnerability in these cells. Collectively, these experiments demonstrate that NOLC1 and UNRIP (through SMN) act synergistically in the Cajal body to assemble snoRNPs and provide the molecular explanation for the synthetic interaction between UNRIP and TP53. 


\section{DISCUSSION}

Our data are consistent with a model in which snoRNP assembly in the Cajal body is the process in which TP53 and UNRIP genetically interact (Fig. 5e). p53 regulates NOLC1 steady-state levels and UNRIP contributes to nuclear SMN import. NOLC1 and SMN support snoRNP assembly in the Cajal bodies and their localization there is mutually dependent. This effect seems to be preferentially important for the box H/ACA snoRNPs. Thus, decreased NOLC1 levels in the knockout cells sensitizes them to the amount of nuclear SMN, which explains their increased dependency on UNRIP for efficient growth and proliferation. This model is substantiated by findings in other model organisms. NOLC1 preferentially affects the box H/ACA snoRNPs ${ }^{46,52}$, and the gradual loss of pseudouridines in rRNA correlates with decreased proliferation ${ }^{50}$. Furthermore, in Drosophila, depletion of NOLC1 expression levels by RNAi leads to development of a Minute phenotype ${ }^{53}$ and in humans the loss of TCOF1, the paralogue of NOLC1, leads to development of Treacher-Collins syndrome ${ }^{54}$, both conditions caused by a loss of ribosome functions. All of these observations show that the rate of cellular proliferation can be adjusted by the rate of ribosome biogenesis.

Our finding that continuous expression of NOLC1 under normal growth conditions is attuned by $\mathrm{p} 53$ implies that $\mathrm{p} 53$ exerts its influence over the ribosome biogenesis pathway; it adjusts the cellular growth and acts in a positive feedback loop of the RP-Hdm2-p53 pathway ${ }^{26}$ to limit ribosome assembly and cellular proliferation under stress conditions. In accordance, p53 upregulation leads to NOLC1 downregulation. Interestingly, this repression seems to be mediated through vacation of $\mathrm{p} 53$ from its binding sites in the NOLC1 promoter, possibly due to competition with other transcription factors or large intergenic non-coding RNA (ref. 55). This so far uncharacterized homeostatic function of $\mathrm{p} 53$ provides yet another mechanism for synchronization of the optimal cellular growth with the environment, which is sensed by p53. As a consequence, p53-negative cells become unable to maintain appropriate growth rates and become vulnerable to interference with specific other processes, such as snoRNP assembly.

\section{METHODS}

Methods and any associated references are available in the online version of the paper at http://www.nature.com/naturecellbiology

Note: Supplementary Information is available on the Nature Cell Biology website

\section{ACKNOWLEDGEMENTS}

We would like to thank all members of the Buchholz laboratory for discussions and sharing reagents. We thank A. K. Heninger for help with carrying out the screen, V. Surendranath for esiRNA design, M. Theis, S. Rose and A. Weise for esiRNA production and I. Poser for assistance with BAC cell line generation. This work was supported by the Max Planck Society, the German Federal Ministry of Education and Research grants Go-Bio (0315105), DiGtoP (01GS0859) and the DFG grant SFB655.

\section{AUTHOR CONTRIBUTIONS}

D.B.K., M.S., N.C.H., M.J. and K.M.N. carried out experiments, M.P-R. analysed data, A.S., M.M. and F.B. planned the project and D.B.K. and F.B. wrote the manuscript.

\section{COMPETING FINANCIAL INTERESTS}

The authors declare no competing financial interests.

Published online at http://www.nature.com/naturecellbiology

Reprints and permissions information is available online at http://www.nature.com/ reprints
1. Levine, A. J. \& Oren, M. The first 30 years of p53: growing ever more complex. Nat. Rev. Cancer 9, 749-758 (2009).

2. Toledo, F. \& Wahl, G. M. Regulating the p53 pathway: in vitro hypotheses, in vivo veritas. Nat. Rev. Cancer 6, 909-923 (2006).

3. Riley, T., Sontag, E., Chen, P. \& Levine, A. Transcriptional control of human p53-regulated genes. Nat. Rev. Mol. Cell Biol. 9, 402-412 (2008).

4. Olovnikov, I. A., Kravchenko, J. E. \& Chumakov, P. M. Homeostatic functions of the p53 tumor suppressor: regulation of energy metabolism and antioxidant defense. Semin. Cancer Biol. 19, 32-41 (2009).

5. Matoba, S. et al. p53 regulates mitochondrial respiration. Science $\mathbf{3 1 2}$, 1650-1653 (2006).

6. Sablina, A. A. et al. The antioxidant function of the p53 tumor suppressor. Nature Med. 11, 1306-1313 (2005).

7. Bensaad, K. et al. TIGAR, a p53-inducible regulator of glycolysis and apoptosis. Cell 126, 107-120 (2006).

8. Kawamura, T. et al. Linking the p53 tumour suppressor pathway to somatic cell reprogramming. Nature 460, 1140-1144 (2009).

9. Hu, W., Feng, Z., Teresky, A. K. \& Levine, A. J. p53 regulates maternal reproduction through LIF. Nature 450, 721-724 (2007).

10. Beltrao, P., Cagney, G. \& Krogan, N. J. Quantitative genetic interactions reveal biological modularity. Cell 141, 739-745 (2010).

11. Kaelin, W. G. The concept of synthetic lethality in the context of anticancer therapy. Nat. Rev. Cancer 5, 689-698 (2005).

12. Luo, J. et al. A genome-wide RNAi screen identifies multiple synthetic lethal interactions with the ras oncogene. Cell 137, 835-848 (2009).

13. Scholl, C. et al. Synthetic lethal interaction between oncogenic KRAS dependency and STK33 suppression in human cancer cells. Cell 137, 821-834 (2009).

14. MacKeigan, J.P., Murphy, L. O. \& Blenis, J. Sensitized RNAi screen of human kinases and phosphatases identifies new regulators of apoptosis and chemoresistance. Nat. Cell Biol. 7, 591-600 (2005).

15. Whitehurst, A. W. et al. Synthetic lethal screen identification of chemosensitizer loci in cancer cells. Nature 446, 815-819 (2007).

16. Bommi-Reddy, A. et al. Kinase requirements in human cells: III. Altered kinase requirements in VHL-/- cancer cells detected in a pilot synthetic lethal screen. Proc. Natl Acad. Sci. USA 105, 16484-16489 (2008).

17. Bunz, F. et al. Requirement for $\mathrm{p} 53$ and $\mathrm{p} 21$ to sustain $\mathrm{G} 2$ arrest after DNA damage. Science 282, 1497-1501 (1998).

18. Vassilev, L. T. et al. In vivo activation of the p53 pathway by small-molecule antagonists of MDM2. Science 303, 844-848 (2004).

19. Shvarts, A. et al. MDMX: a novel p53-binding protein with some functional properties of MDM2. EMBO J. 15, 5349-5357 (1996).

20. Kittler, R. et al. Genome-scale RNAi profiling of cell division in human tissue culture cells. Nat. Cell Biol. 9, 1401-1412 (2007).

21. Theis, M. et al. Comparative profiling identifies $\mathrm{C} 13$ orf3 as a component of the Ska complex required for mammalian cell division. EMBO J. 28, 1453-1465 (2009).

22. Slabicki, M. et al. A genome-scale DNA repair RNAi screen identifies SPG48 as a novel gene associated with hereditary spastic paraplegia. PLoS Biol. 8, e1000408 (2010)

23. Matthews, L. et al. Reactome knowledgebase of human biological pathways and processes. Nucliec Acids Res. 37, D619-D622 (2009).

24. Jensen, L. et al. STRING 8-a global view on proteins and their functional interactions in 630 organisms. Nucliec Acids Res. 37, D412-D416 (2009).

25. Zhang, X. D. et al. Robust statistical methods for hit selection in RNAinterference high-throughput screening experiments. Pharmacogenomics 7, 299-309 (2006).

26. Deisenroth, C. \& Zhang, Y. Ribosome biogenesis surveillance: probing the ribosomal protein-Mdm2-p53 pathway. Oncogene 29, 1-8 (2010).

27. Fromont-Racine, M., Senger, B., Saveanu, C. \& Fasiolo, F. Ribosome assembly in eukaryotes. Gene 313, 17-42 (2003).

28. Sur, S. et al. A panel of isogenic human cancer cells suggests a therapeutic approach for cancers with inactivated p53. Proc. Natl Acad. Sci. USA 106, 3964-3969 (2009).

29. Datta, P. K., Chytil, A., Gorska, A. E. \& Moses, H. L. Identification of STRAP, a novel WD domain protein in transforming growth factor- $\beta$ signaling. J. Biol. Chem. 273, 34671-34674 (1998).

30. Carissimi, C. et al. Unrip is a component of SMN complexes active in snRNP assembly. FEBS Lett. 579, 2348-2354 (2005).

31. Kittler, R. et al. RNA interference rescue by bacterial artificial chromosome transgenesis in mammalian tissue culture cells. Proc. Natl Acad. Sci. USA 102, 2396-2401 (2005).

32. Poser, I. et al. BAC TransgeneOmics: a high-throughput method for exploration of protein function in mammals. Nat. Methods 5, 409-415 (2008).

33. Battle, D. et al. The SMN complex: an assembly machine for RNPs. Cold Spring Harb. Symp. Quant. Biol. 71, 313-320 (2006).

34. Hao, L. T. et al. Absence of gemin5 from SMN complexes in nuclear Cajal bodies. BMC Cell Biol. 8, 28 (2007).

35. Narayanan, U., Achsel, T., Lührmann, R. \& Matera, A. G. Coupled in vitro import of $U$ snRNPs and SMN, the spinal muscular atrophy protein. Mol. Cell 16, 223-234 (2004). 
36. Fischer, U., Sumpter, V., Sekine, M., Satoh, T. \& Luhrmann, R. Nucleo-cytoplasmic transport of $U$ snRNPs: definition of a nuclear location signal in the $\mathrm{Sm}$ core domain that binds a transport receptor independently of the m3G cap. EMBO J. 12, 573-583 (1993).

37. Hamm, J., Darzynkiewicz, E., Tahara, S. M. \& Mattaj, I. W. The trimethylguanosine cap structure of U1 snRNA is a component of a bipartite nuclear targeting signal. Cell 62, 569-577 (1990).

38. Hubner, N. C. et al. Quantitative proteomics combined with BAC TransgeneOmics reveals in vivo protein interactions. J. Cell Biol. 189, 739-754 (2010).

39. Ong, S. E. et al. Stable isotope labelling by amino acids in cell culture, SILAC, as a simple and accurate approach to expression proteomics. Mol. Cell Proteomics 1 376-386 (2002)

40. Smeenk, L. et al. Characterization of genome-wide p53-binding sites on stress response. Nucleic Acids Res. 17, 3639-3654 (2008).

41. Meier, U. T. \& Blobel, G. Nopp140 shuttles on tracks between nucleolus and cytoplasm. Cell 70, 127-138 (1992).

42. Pai, C. Y., Chen, H. K., Sheu, H. L. \& Yeh, N. H. Cell-cycle-dependent alterations of a highly phosphorylated nucleolar protein p130 are associated with nucleologenesis. J. Cell Sci. 108, 1911-1920 (1995).

43. Isaac, C., Yang, Y. \& Meier, U. T. Nopp140 functions as a molecular link between the nucleolus and the coiled bodies. J. Cell Biol. 142, 319-329 (1998).

44. Renvoise, B. et al. The loss of the snoRNP chaperone Nopp140 from Cajal bodies of patient fibroblasts correlates with the severity of spinal muscular atrophy. Hum. Mol. Genet. 18, 1181-1189 (2009).

45. Pellizzoni, L., Baccon, J., Charroux, B. \& Dreyfuss, G. The survival of motor neurons (SMN) protein interacts with the snoRNP proteins fibrillarin and GAR1. Curr. Biol. 11, 1079-1088 (2001).
46. Yang, Y. et al. Conserved composition of mammalian box H/ACA and box C/D small nucleolar ribonucleoprotein particles and their interaction with the common factor Nopp140. Mol. Biol. Cell 11, 567-577 (2000).

47. Wang, C., Query, C. C. \& Meier, U. T. Immunopurified small nucleolar ribonucleoprotein particles pseudouridylate rRNA independently of their association with phosphorylated Nopp140. Mol. Cell Biol. 22, 8457-8466 (2002).

48. Verheggen, C. et al. Box C/D small nucleolar RNA trafficking involves small nucleolar RNP proteins, nucleolar factors and a novel nuclear domain. EMBO J. 20, 5480-5490 (2001).

49. Darzacq, X. et al. Stepwise RNP assembly at the site of H/ACA RNA transcription in human cells. J. Cell Biol. 173, 207-218 (2006).

50. King, T. H., Liu, B., McCully, R. R. \& Fournier, M. J. Ribosome structure and activity are altered in cells lacking snoRNPs that form pseudouridines in the peptidyl transferase center. Mol. Cell 11, 425-435 (2003).

51. Lafontaine, D. L., Bousquet-Antonelli, C., Henry, Y., Caizergues-Ferrer, M. \& Tollervey, D. The box H+ACA snoRNAs carry Cbf5p, the putative rRNA pseudouridine synthase. Genes Dev. 12, 527-537 (1998).

52. Yang, Y. \& Meier, U. T. Genetic interaction between a chaperone of small nucleolar ribonucleoprotein particles and cytosolic serine hydroxymethyltransferase. J. Biol. Chem. 278, 23553-23560 (2003).

53. Cui, Z. \& DiMario, P. J. RNAi knockdown of Nopp140 induces Minute-like phenotypes in Drosophila. Mol. Biol. Cell 18, 2179-2191 (2007).

54. Isaac, C. et al. Characterization of the nucleolar gene product, treacle, in Treacher Collins syndrome. Mol. Biol. Cell 11, 3061-3071 (2000).

55. Huarte, M. et al. A large intergenic noncoding RNA induced by p53 mediates global gene repression in the p53 response. Cell 143, 409-419 (2010). 


\section{METHODS}

Cell culture, constructs and reagents. HCT116 and RKO (wild-type and TP53 knockout) cells (kind gift from B. Vogelstein, Sidney Kimmel Comprehensive Cancer Center, Johns Hopkins University, Baltimore, USA) were maintained in $4.5 \mathrm{mg} \mathrm{ml}^{-1}$ glucose DMEM, supplemented with $10 \%$ FBS (Invitrogen), $100 \mathrm{U} \mathrm{ml}^{-1}$ penicillin and $100 \mu \mathrm{g} \mathrm{ml}{ }^{-1}$ streptomycin (Gibco-Invitrogen). Enhanced GFP (EGFP)-tagged nuclear localization signal (NLS) protein and mRed-NLS were cloned into pIRESpuro3 plasmid (Clontech) and stable cell lines were selected with $1 \mu \mathrm{g} \mathrm{ml}^{-1}$ puromycin (Sigma-Aldrich). BAC clones RP11-413P14 and RP24191F17 encoding human and mouse UNRIP genes respectively were obtained from BACPAC resource. They were tagged as previously described ${ }^{32}$ and stable cell lines were selected on the basis of their neomycin $\left(0.5 \mathrm{mg} \mathrm{ml}^{-1}\right.$, Invitrogen) or blasticidine ( $5 \mu \mathrm{g} \mathrm{ml}^{-1}$, Invivogen) resistance.

The following antibodies and proteins were used: anti-GFP (Roche, MPI-CBG, 1:5,000), anti- $\alpha$-tubulin (MPI-CBG, 1:50,000), anti-UNRIP (sc-14552, 1:1,000), anti-SMN1 (ab5831, 1:1,000), anti-Gemin2 (ab6084, 1:1,000), anti-SmB (sc25372, 1:1,000), anti-COIL (1:500; ref. 56; sc-32860, 1:500), anti-PCNA (sc-9857, 1:1,000), anti-p53 (DO-1), anti-NOLC1 (sc-101101, 1:1,000), anti-histone3 (ab1791, 1:10,000), anti-p21/WAF1 (sc-6246, 1:1,000), camptothecin (Sigma-Aldrich), bleocin (Merck Bioscience) and Nutlin-3a (Sigma-Aldrich). p21-luc (ref. 57), 3TP-luc (ref. 58), pCS2-Smad2 (ref. 59) and pRK5-T202D (ref. 60) were obtained from the Addgene repository and the Smad reporter kit was from SuperArray. The GFP-NAF1-expressing plasmid was a kind gift from U. T. Meier ${ }^{49}$ (Department of Anatomy and Structural Biology, Albert Einstein College of Medicine, New York, USA). The NOLC1 promoter sequence was amplified and cloned into pGL4.10 (Promega) reporter plasmid. UNRIP Mission shRNA-expressing plasmids (shRNA1- NM_007178.2-981slc1 and shRNA- NM_007178.2-981slc1) were from Sigma-Aldrich.

esiRNA preparation and library construction were carried out as previously described $^{61}$. Typically, HCT116 cells were transfected in 384 -well plates with $25 \mathrm{ng}$ esiRNA and $0.25 \mu \mathrm{l}$ Oligofectamine (Invitrogen) in $10 \mu \mathrm{l} \mathrm{OptiMem} \mathrm{(Invitrogen).} \mathrm{For}$ transfections in other plate formats the esiRNA amounts were scaled according to the surface area of the wells.

High-throughput screen, data analysis and hits validation. A total of 1,000 wild-type and 1,000 knockout HCT116 cells, in $100 \mu \mathrm{l}$ of cell suspension, were reverse transfected in black 384-well tissue culture plates (Greiner). Plates were sealed with breathable foil (Corning) and incubated for $96 \mathrm{~h}$. Cells were fixed and four images per well were acquired using a ScanR screening station (Olympus). The number of wild-type and knockout cells was exported with the ScanR analysis software. The screen was conducted in two replicates. Each plate contained twelve negative (luciferase) and four positive (HDMX) control esiRNAs. The cell numbers (Supplementary Table S1) for each esiRNA knockdown (each well position) were calculated for the wild-type and knockout cells separately:

$$
X_{i, p}=\frac{X_{i, p}(\text { raw })}{\bar{X}} \times \overline{X_{p}}
$$

$X_{i, p}$ is the cell number of well $i$ and plate $p, X_{i, p}($ raw $)$ is the cell number extracted from the image analysis, $\bar{X}$ is the cell number of well $i$ averaged over all plates and $\overline{X_{p}}$ is the average cell number of all wells in plate $p$. The $X_{i, p}$ values were used to calculate the wild-type/knockout ratio. The $z$ scores of the cell numbers, showing how many standard deviations $\left(\sigma_{p}\right)$ the observed cell number $\left(X_{i, p}\right)$ is different from the mean cell number $\left(\overline{X_{p}}\right)$, is calculated in a plate-wise manner.

$$
z=\frac{X_{i, p}-\overline{X_{p}}}{\sigma_{p}}
$$

A viability phenotype was assigned to each esiRNA with $z$ scores for both wild-type and knockout cells lower than -2 (or -1.5$)$ in any of the technical replicates of the screen. Using a quartile-based threshold we divide the $\log _{2}$ (wild - type/knockout) into three phenotypic categories in a plate-wise manner:

$$
\begin{array}{lll}
\text { Decreased wild-type/knockout ratio } & -1 & X_{i}<Q_{1}-2 c\left(Q_{2}-Q_{1}\right) \\
\text { No change in wild-type/knockout ratio } & 0 & X_{i} \in\left(Q_{1}-2 c\left(Q_{2}-Q_{1}\right) ;\right. \\
& & \left.Q_{3}+2 c\left(Q_{3}-Q_{2}\right)\right) \\
\text { Increased wild-type/knockout ratio } & 1 & X_{i}>Q_{3}+2 c\left(Q_{3}-Q_{2}\right)
\end{array}
$$

where $X_{i}$ denotes the $\log _{2}$ (wild - type/knockout) for a given esiRNA, Q1, Q2 and Q3 are the first, second and third quartile values of the corresponding plate and $c$ is a constant, which can be chosen depending on the targeted error rate under the null hypothesis (for an error rate of $0.05 c=0.9529$; ref. 25). The primary hits were selected on the basis of the condition of scoring as 1 or -1 in both technical replicates. For the validation screens and secondary esiRNAs, $z$ scores (always of wild-type/knockout ratio) are calculated on the basis of the variation of negative control transfections (luciferase). From each set of controls and treatment samples up to one outlier was removed on the basis of Grubb's outlier removal test. Phenotypes were considered validated when they were statistically significant according to Student's $t$-test $(P<0.05)$.

Time-lapse analysis. HCT116 wild-type and knockout cells were mixed in a 1:1 ratio and reverse transfected with esiRNAs. At $24 \mathrm{~h}$ post-transfection, the cells were imaged for another $72 \mathrm{~h}$ with an image acquired every $30 \mathrm{~min}$ with a Scan R imaging system (Olympus). Doubling times were calculated from exponential fits to the growth curves.

Colony formation assay. RNAi-treated cells were trypsinized $24 \mathrm{~h}$ posttransfection; 2,000 cells were seeded in six-well plates and cultivated for a week. Colonies were fixed with paraformaldehyde and stained with $0.05 \%$ crystal violet (Sigma). The number of colonies was analysed with Cell Profiler software ${ }^{62}$ or absorbtion at $540 \mathrm{~nm}$ was determined after methanol extraction.

Luciferase assay. All firefly constructs were mixed in a 40:1 ratio with a constitutive Renilla-expressing luciferase construct, pGL4.73 (Promega). The co-transfection of esiRNA and plasmid DNA was carried out with SureFECT transfection reagent (SuperArray). Luciferase luminescence was measured with the Dual-Glo Luciferase Assay System (Promega) on a GENios pro plate reader (Tecan). The firefly signal was normalized to the Renilla signal and the average and standard deviation of at least three independent experiments are presented.

Immunoprecipitation, mass-spectrometry and QUBIC experiments. Immunoprecipitation reactions were carried out with extracts from cells expressing LAP-tagged proteins and subjected to shotgun mass spectrometry (MS) as previously described ${ }^{21,32}$. Briefly, LAP-tagged proteins were immunoprecipitated with anti-GFP antibody (MPI-CBG) coupled to G-protein Sepharose (GE Healthcare). Glycine-eluted complexes were digested in solution using trypsin, and the resulting peptides were analysed by shotgun liquid chromatography-MS/MS on an LTQ Orbitrap (Thermo Fisher Scientific) ${ }^{63}$. Proteins identified in more than $15 \%$ of more than 200 immunoprecipitations from unrelated bait proteins were considered as common background proteins and were excluded from further analysis. For QUBIC, HCT116 wild-type or knockout cells expressing human UNRIP-LAP were SILAC-labelled ${ }^{64}$ in DMEM media, supplemented with heavy- $\left({ }^{13} \mathrm{C}^{15} \mathrm{~N}\right)$ or light- $\left({ }^{12} \mathrm{C}^{14} \mathrm{~N}\right)$ isotope-labelled arginine and lysine. After seven days, extracts were prepared from $2 \times 10^{7}$ cells and complexes were precipitated with an anti-GFP antibody (Miltenyi Biotec). The eluted proteins were mixed in a 1:1 ratio and subjected to mass spectrometry ${ }^{38}$. The experiment was repeated with extracts from reverse labelled cells.

qRT-PCR. At $48 \mathrm{~h}$ post-transfection, total RNA was isolated with an RNeasy Mini Kit (Qiagen) and 500 ng was reverse transcribed with SuperScript III reverse transcriptase (Invitrogen). Quantitative PCR reactions were carried out with the SYBR Green qPCR kit (Abgene) on an MX P3000 qPCR machine (Stratagene) according to the manufacturer's recommendations. Relative mRNA levels were calculated according to the $\Delta \Delta \mathrm{Ct}$ threshold cycle method and normalized to the luciferase control; GAPDH was used as an internal loading control.

Immunofluorescence microscopy. Cells were seeded on coverslips in 24-well plates, transfected $24 \mathrm{~h}$ later and immunostained as previously described ${ }^{21}$. Images were acquired on an Axioplan II microscope (Zeiss) operated by MetaMorph (Molecular Devices) or on an Olympus IX70 microscope (Olympus) equipped with a DeltaVision RT imaging system. For nuclear foci scoring, $z$ stacks were acquired, deconvolved and projected into a single plane using softWoRx software. Contrast levels were manually adjusted and at least 200-300 cell nuclei were manually scored per sample. The distributions were compared using Student's two-tailed $t$-test.

Cell fractionation. Cell fractionation experiments were carried out with the ProteoExtract Subcellular Proteome Extraction Kit (Calbiochem) according to the manufacturer's recommendations.

p53 ChIP. HCT116 cells $\left(2 \times 10^{7}\right)$ were treated with $5 \mu \mathrm{M}$ Nutlin-3 $\alpha$ for $4 \mathrm{~h}$ and p53 was precipitated with $4 \mu \mathrm{g}$ DO-1 antibody as described previously ${ }^{65}$. The fold change was calculated as the ratio of the percentage inputs from wild-type and knockout cells.

snRNP assembly assay. The snRNP immunoprecipitation protocol was modified from ref. 56. Briefly, at $36 \mathrm{~h}$ post-transfection the medium was replaced with phosphate-free DMEM medium (Gibco, Invitrogen) supplemented with $20 \mu \mathrm{Ci} \mathrm{ml}^{-132} \mathrm{P}$-orthophosphate. After $16 \mathrm{~h}$ incubation, the cells were scraped into NET-2 buffer (50 mM Tris- $\mathrm{HCl}$ at $\mathrm{pH} 7.5,150 \mathrm{mM} \mathrm{NaCl}$ and $0.05 \% \mathrm{NP}-40$ ), sonicated and centrifuged to remove debris and unlysed cells. The cell extract 
was incubated with Y12- (recognizes the Sm ring ${ }^{66}$ ) or anti-TMG- (recognizes the tri-methylated cap; NA02 Merk Bioscience) conjugated Gammabind G beads (GE Healthcare). Bound RNA was extracted with phenol/chloroform and precipitated overnight with ethanol. The RNA samples were resolved on a denaturating $7 \mathrm{M}$ urea $10 \%$ PAGE gel and imaged with a phosphoimager (Fujifilm BAS-1800II).

snoRNP assembly assay. Cells were seeded in six-well plates and subjected to RNAi for $48 \mathrm{~h}$, followed by overexpression of GFP-NAF1 for a further $24 \mathrm{~h}$. A quantity of $100 \mu \mathrm{g}$ total cellular extract (prepared in NET-2 buffer) was incubated with $50 \mu \mathrm{l}$ GFP-Trap beads (ChromoTek) and associated pre-snoRNAs were phenol-purified, DNase-I-treated and reverse transcribed with specific pre-snoRNA primers.

Metabolic labelling and pulse-chase assays. rRNA cleavage maturation was investigated in ${ }^{32} \mathrm{P}$-orthophosphate labelled cells. Briefly, before labelling, the cells were incubated in P-free DMEM media (Invitrogen) for $1 \mathrm{~h}$, labelled with $20 \mu \mathrm{Ci} \mathrm{ml}^{-1}{ }^{32} \mathrm{P}$-orthophosphate for $1 \mathrm{~h}$ and chased with normal growth media for $30 \mathrm{~min}$. Total RNA was extracted with Tryzol (Invitrogen), and $5 \mu \mathrm{g}$ was resolved on a denaturing agarose gel and autoradiographed for $24 \mathrm{~h}$. For rRNA methylation, the cells were incubated in methionine-free MEM media (Invitrogen) for $15 \mathrm{~min}$ before being labelled with $50 \mu \mathrm{Ci} \mathrm{ml}^{-1} \mathrm{~L}$-[methyl-3H]-methionine for $30 \mathrm{~min}$ and chased with $0.3 \mathrm{mg} \mathrm{ml}^{-1}$ methionine-containing DMEM for $30 \mathrm{~min}$. Total RNA was extracted with Tryzol (Invitrogen), and $5 \mu \mathrm{g}$ was resolved on a denaturing agarose gel, transferred to a nitrocellulose membrane and autoradiographed for $3-5$ days.

RNA fluorescence in situ hybridization. The mature snoRNAs were amplified from a total RNA sample and the PCR products were used as a template for in vitro RNA transcription with SP6 polymerase (MPI-CBG) in the presence of FITC (fluorescein isothiocyanate)-conjugated UTP (Invitogen). The hybridization was conducted as described previously ${ }^{67}$.

CMCT assay. CMCT ( $N$-cyclohexyl- $N^{\prime}-\beta-(4-m e t h y l m o r p h o l i n i u m)$ ethylcarbodiimide p-tosylate) modification of RNA was carried out as described previously ${ }^{68}$. Briefly, total RNA from RNAi-depleted HCT116 cells was extracted with Trizol (Invitrogen) and $10 \mu \mathrm{g}$ was CMCT modified. A quantity of $0.5 \mu \mathrm{g}$ modified RNA was used in primer extension reactions with SuperScript III reverse transcriptase (Invitrogen) and the primers 28S_1,28S_2, 18S_1and18S_2. The products were resolved on denaturing 10\% PAGE and quantified on a phosphoimager (Fujifilm
BAS-1800II). Equal loading of the lanes was ensured by northern blot analysis of the $28 \mathrm{~S}$ rRNA.

All primers used in this study are listed in Supplementary Table S4.

56. Pettersson, I., Hinterberger, M., Mimori, T., Gottlieb, E. \& Steitz, J. A. The structure of mammalian small nuclear ribonucleoproteins. Identification of multiple protein components reactive with anti-(U1)ribonucleoprotein and anti-Sm autoantibodies. J. Biol. Chem. 259, 5907-5914 (1984).

57. el-Deiry, W. S. et al. WAF1, a potential mediator of p53 tumor suppression. Cell 75, 817-825 (1993).

58. Wrana, J. L. et al. TGF $\beta$ signals through a heteromeric protein kinase receptor complex. Cell 71, 1003-1014 (1992).

59. Hata, A., Lo, R. S., Wotton, D., Lagna, G. \& Massague, J. Mutations increasing autoinhibition inactivate tumour suppressors Smad2 and Smad4. Nature 388, 82-87 (1997).

60. Feng, X. H. \& Derynck, R. Ligand-independent activation of transforming growth factor (TGF) $\beta$ signalling pathways by heteromeric cytoplasmic domains of TGF- $\beta$ receptors. J. Biol. Chem. 271, 13123-13129 (1996).

61. Kittler, R. et al. Genome-wide resources of endoribonuclease-prepared short interfering RNAs for specific loss-of-function studies. Nat. Methods 4, 337-344 (2007).

62. Lamprecht, M. R., Sabatini, D. M. \& Carpenter, A. E. CellProfiler: free, versatile software for automated biological image analysis. Biotechniques 42, 71-75 (2007).

63. Junqueira, M. et al. Separating the wheat from the chaff: unbiased filtering of background tandem mass spectra improves protein identification. J. Proteome Res. 7, 3382-3395 (2008).

64. Ong, S. E. \& Mann, M. Mass spectrometry-based proteomics turns quantitative. Nat. Chem. Biol. 1, 252-262 (2005).

65. Listerman, I., Bledau, A. S., Grishina, I. \& Neugebauer, K. M. Extragenic accumulation of RNA polymerase II enhances transcription by RNA polymerase III. PLoS Genet. 3, e212 (2007).

66. Almeida, F., Saffrich, R., Ansorge, W. \& Carmo-Fonseca, M. Microinjection of anti-coilin antibodies affects the structure of coiled bodies. J. Cell Biol. 142, 899-912 (1998).

67. Chaumeil, J., Augui, S., Chow, J. C. \& Heard, E. Combined immunofluorescence, RNA fluorescent in situ hybridization, and DNA fluorescent in situ hybridization to study chromatin changes, transcriptional activity, nuclear organization, and X-chromosome inactivation. Methods Mol. Biol. 463, 297-308 (2008).

68. Bakin, A. \& Ofengand, J. Four newly located pseudouridylate residues in Escherichia coli $23 \mathrm{~S}$ ribosomal RNA are all at the peptidyltransferase center: analysis by the application of a new sequencing technique. Biochemistry 32, 9754-9762 (1993). 


\section{cell biology}

DOI: $10.1038 / \mathrm{ncb} 2264$
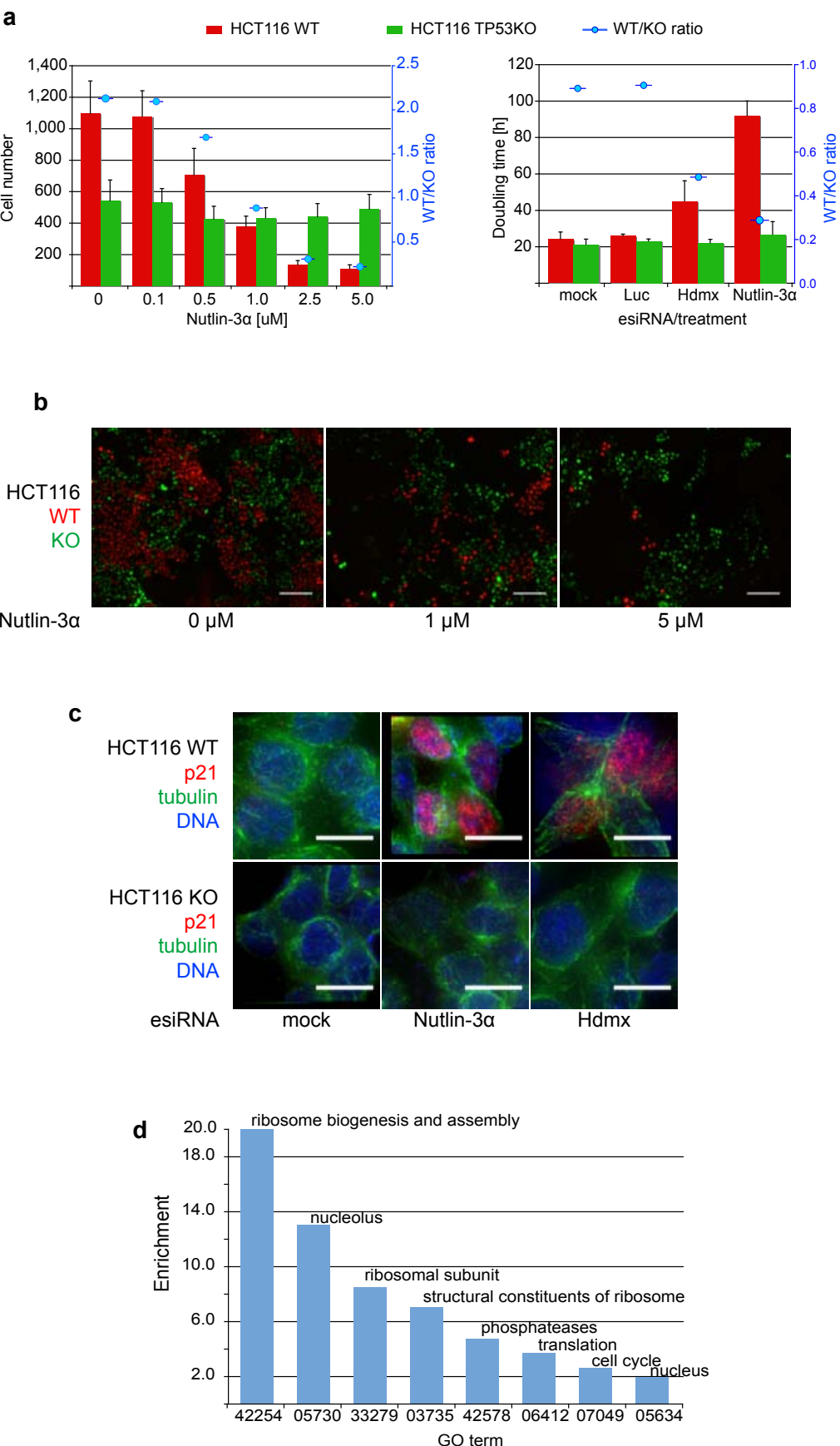

Figure S1 Screening assay evaluation. (a) Left panel - dose-response of WT and KO cell number to different concentrations of Nutlin-3a. WT and $\mathrm{KO}$ cells were mixed in 2:1 ratio and grown for 48 hours in the presence of Nutlin-3a. The left y-axis shows the cell number and the right $y$-axis (blue) represents the WT/KO ratio. Right panel - doubling time of WT and KO cell upon RNAi depletion of control genes or Nutlin-3a treatment (mean \pm s.d., $n=3$ ). (b) Images of mixed population of WT (red) and KO (green) cells co-cultured in the presence of different concentrations of Nutlin-3a, scale bar represents distance of $100 \mu \mathrm{m}$ (mean \pm s.d., $\mathrm{n}=3$ ) (c) Indirect immunofluorescence for p21 WAF1 protein (red) in WT and KO cells after treatment with $1 \mathrm{mM}$ Nutlin-3a or Hdmx depletion. a-tubulin is stained in green and DNA in blue (DAPI), scale bar represents distance of $10 \mu \mathrm{m}$. (d) Gene Ontology terms enrichment analysis of the hits decreasing the WT/KO ratio according to the web-based DAVID bioinformatics resource at the National Institute of Allergy and Infectious Diseases, NIH. Presented are the GO terms with enrichment above 2 . 
a

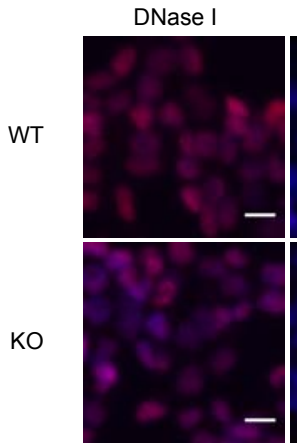

b

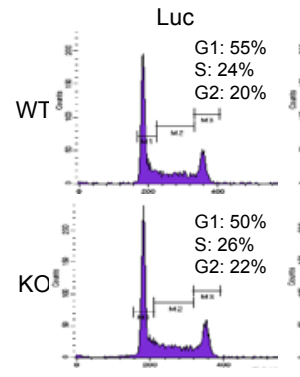

Luc

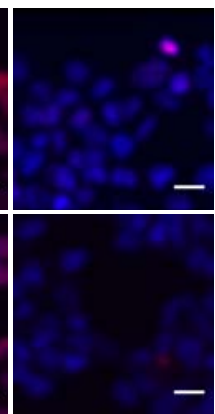

UNRIP
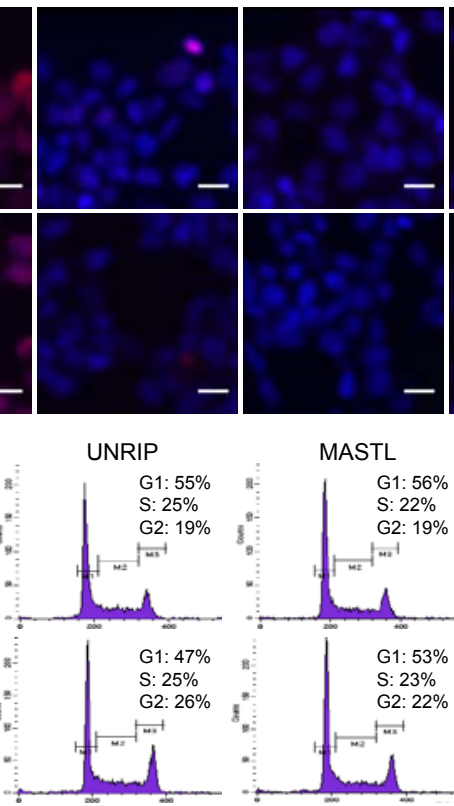

MASTL

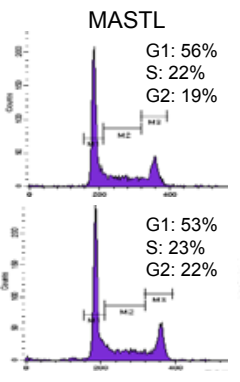

MASTL

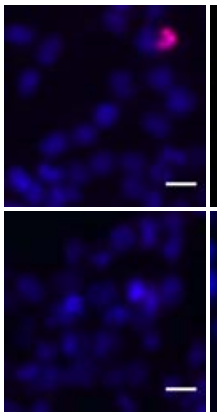

KIAA1344
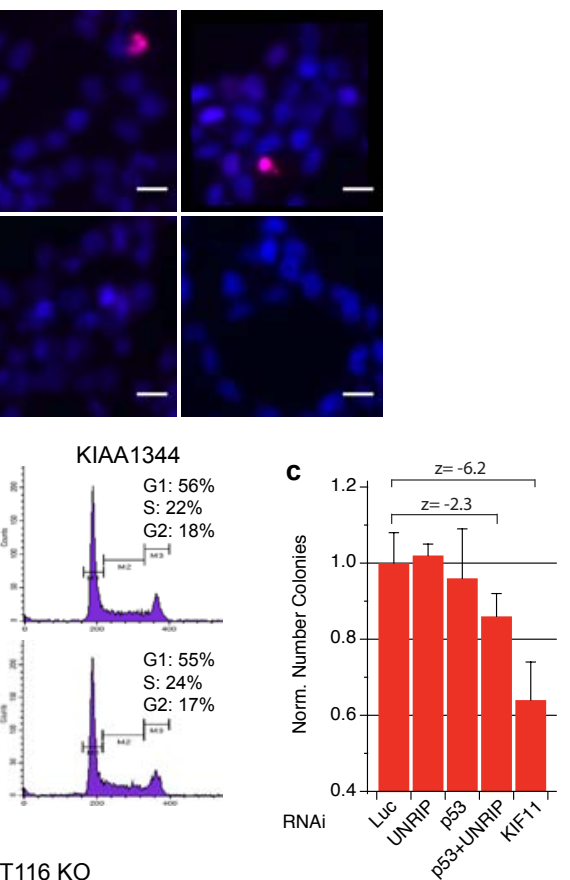

HCT116 WT

HCT116 KO

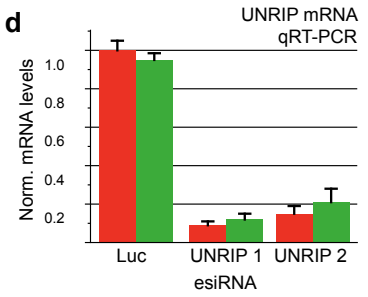

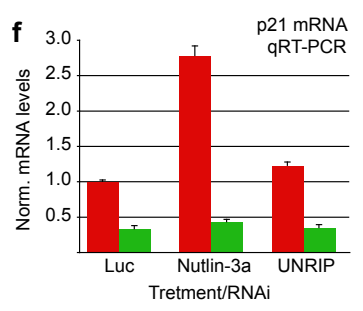

g

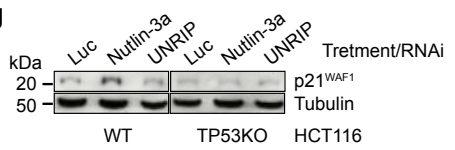

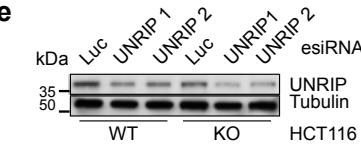

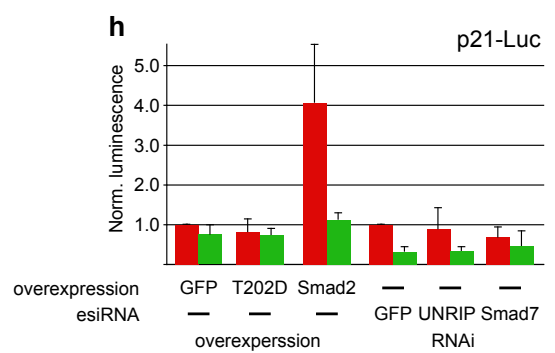

i

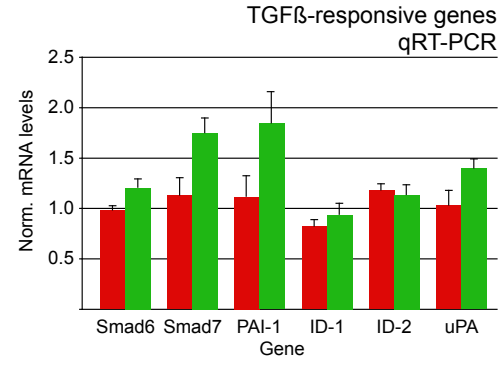

Figure S2 Characterization of the depletion phenotypes of the genes that increase the WT/KO ratio. (a) Hits depletion does not lead to apoptosis induction judged by terminal deoxynucleotidyl transferase dUTP nick end labeling (TUNEL) assay (red signal). DNase I treatment shows the robustness of the assay to detect nicked DNA. Scale bars represents distance of $10 \mu \mathrm{m}$. (b) Hits depletion does not lead to cell cycle arrest judged by the DNA quantity profiles (propidium iodide staining). (c) Colony formation assay in HCT116 WT cells. TP53 and UNRIP co-depletion leads to decreased clonogenic survival (z-score -2.3). KIF11 depletion (z-score -6.2), which gives strong decrease in clonogenic survival, is provided for comparison. Presented is the number of colonies, normalized to luciferase transfection (d,e) Quantification of UNRIP mRNA and protein knockdown with two independent esiRNAs. Fold change over control (luciferase)

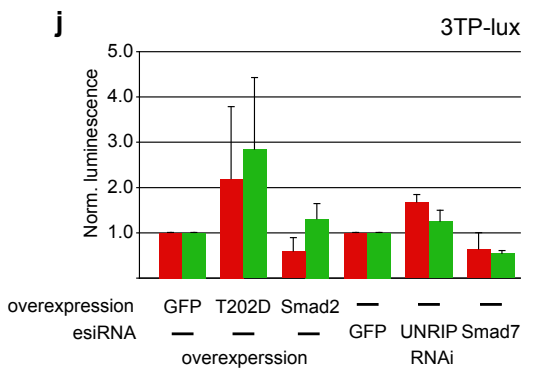

depletion (mean \pm s.d., $n=3$ ). (f, $\mathbf{g})$ Quantification of p21/WAF1 mRNA and protein levels upon UNRIP knockdown. The fold change is calculated over luciferase knockdown in the WT cells (mean \pm s. $d, n=3$ ). Treatment with $1 \mathrm{mM}$ Nutlin-3a for 24 hours is used as a control of p53 activation. (h) p21 WAF1_luc reporter induction upon TGFb signaling activation (overexpression of TbRI-T202D, Smad2) or depletion (UNRIP, Smad7). The values are normalized to GFP overexpression or depletion, respectively. (i) Quantitative RT-PCR analysis of TGFb responsive genes upon UNRIP depletion. Fold change over control (luciferase) depletion (mean \pm s.d., $\mathrm{n}=3$ ). (j) 3TP-lux reporter induction upon TGFb signaling activation (overexpression of TbRI-T202D, Smad2) or depletion (UNRIP, Smad7). The values are normalized to negative controls GFP overexpression or depletion, respectively (mean \pm s.d., $n=3$ ). 
a

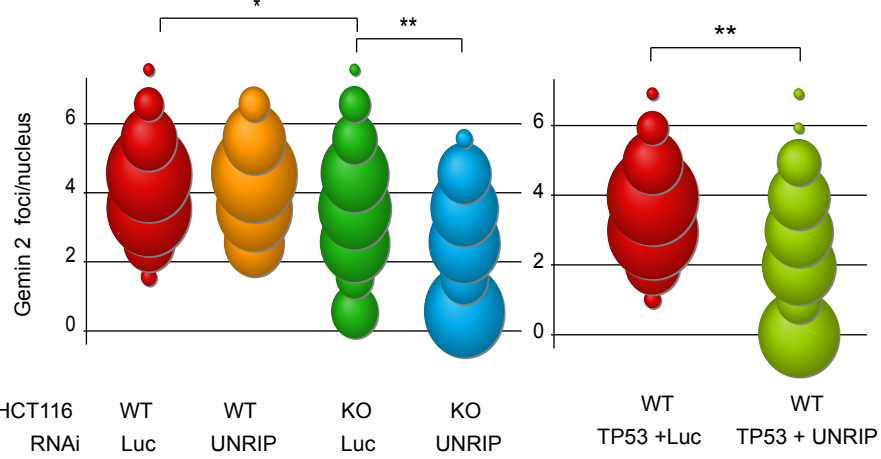

C

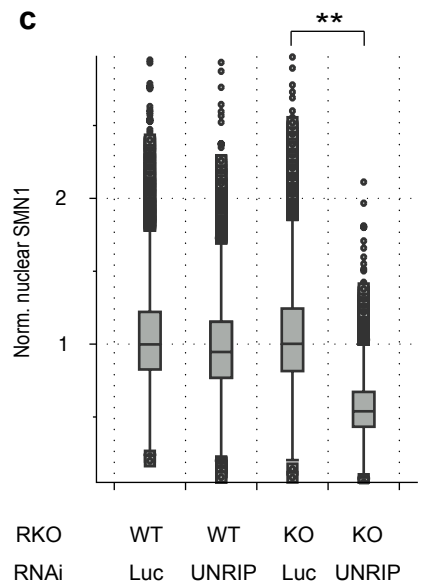

b

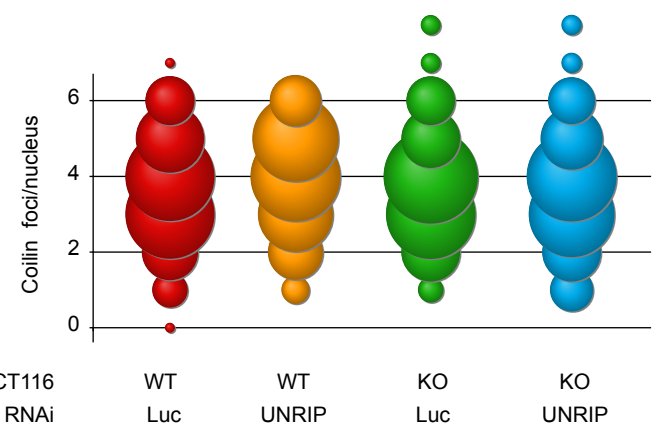

d

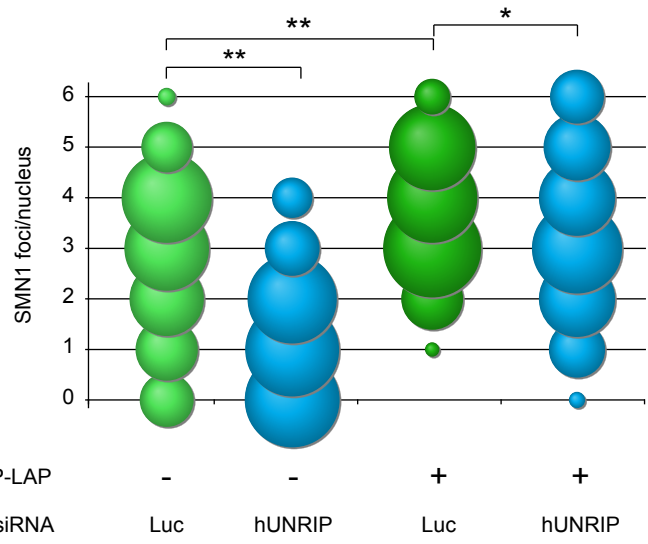

e

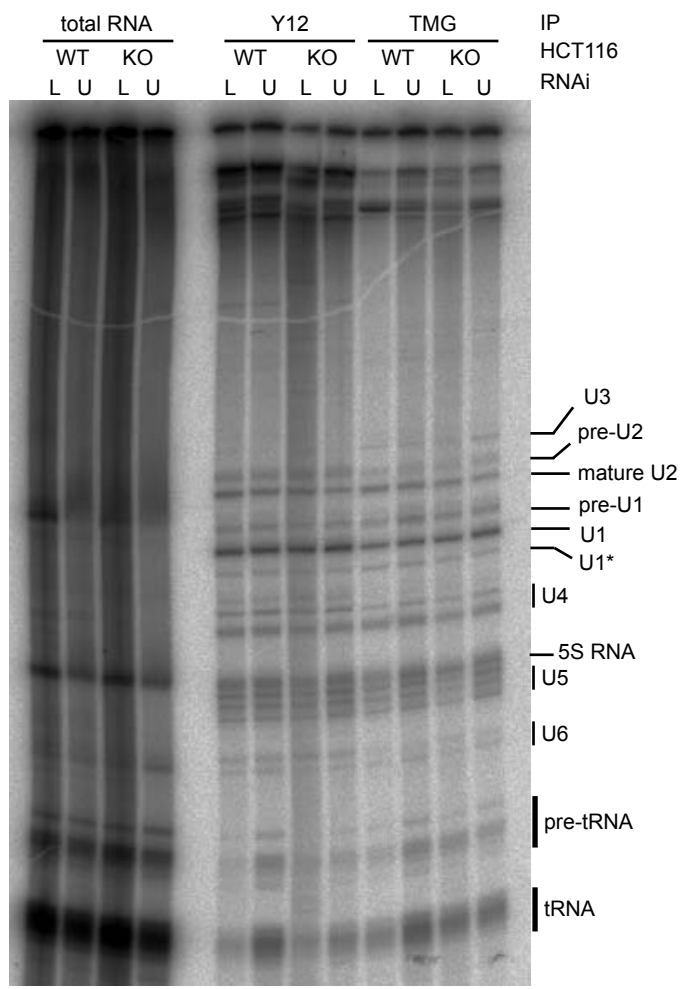

Figure S3 UNRIP depletion influences SMN complex localization but not snRNAP assembly activity. Quantification of Gemin2 (a) and p80/Coilin (b) foci per nucleus upon UNRIP depletion. The sizes of the balls represent the percentage of the cells with the corresponding number of foci. The $p$-values were calculated with two-tailed $t$-test, * $-p$-values $<0.01$, ** $p$-values $<10^{-5}$, counted 200-300 nuclei per condition in three independent experiments. (c) Box plot showing the amount of nuclear SMN1, determined by immunofluorescent staining, in RKO cells upon UNRIP depletion. The median of luciferase depleted RKO WT cells is normalized to 1 . The $p$-values were calculated with two-tailed $t$-test, ${ }^{* *}$ - $p$-values $<0.01$. (d) Expression of mouse UNRIP-LAP in HCT116 KO cells rescues SMN1's localization to the CB upon depletion of the endogenous human UNRIP. (e) UNRIP is dispensable for the snRNP assembly in vivo. HCT116 cells were UNRIP depleted (L- luciferase, U - UNRIP), ${ }^{32}$ P-orthophosphate metabolically labeled and snRNPs, immunoprecipitated with anti-Sm (Y12) or anti-TMG antibody, were analyzed on a denaturing AA-PAGE. U1* - degradation product of U1 SnRNA. 
a

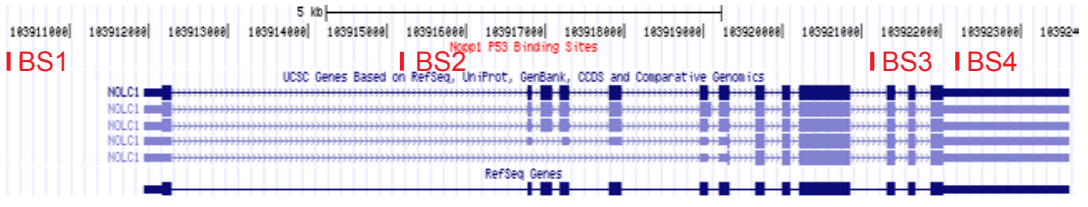

b

Site Sequence

p53 consensus RRRCWWGYYY RRRCWWGYYY

Nolc1 BS1 AGAGAAGCTC ACACTTGTTC

Nolc1 BS2 TGTCTTGCCC AGGCTGGTCT

Nolc1 BS3 AGTTTTGTAC AGACTTGTTT

Nolc1 BS4 AAGCATGCAC CACCATGTCT

$\begin{array}{cc}\begin{array}{c}\text { Start site } \\ \text { relative to TSS } \\ \text { (bp) }\end{array} & \begin{array}{c}\text { p53scan } \\ \text { score }\end{array} \\ -1567 & 5.235 \\ 3254 & 7.302 \\ 9195 & 6.657 \\ 10264 & 6.628\end{array}$

\section{$\mathrm{R}=$ purine $\quad \mathrm{W}=\mathrm{A}$ or $\mathrm{T} \quad \mathrm{Y}=$ pyrimidine}

C

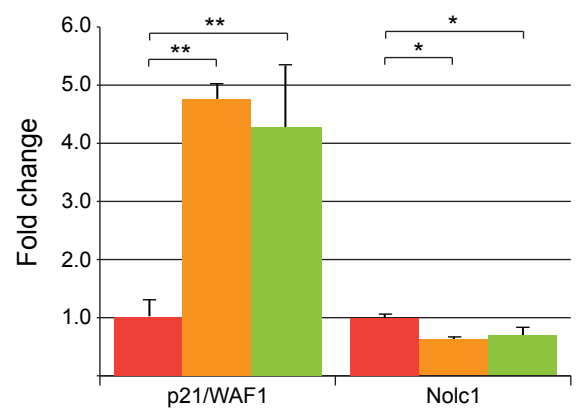

WT/mock
qRT-PCR

WT/Camptothecin
WT/Bleocin d

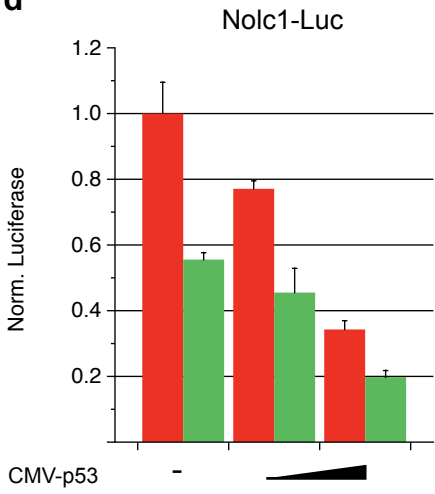

TP53 WT

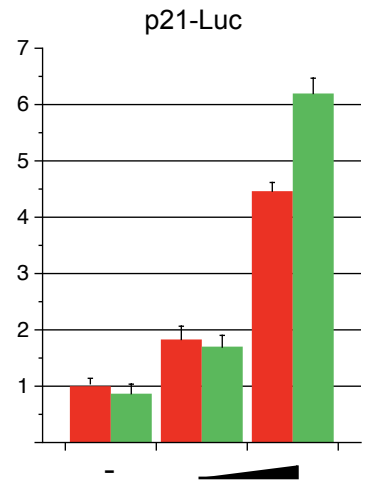

TP53 KO
Figure S4 Description of $\mathrm{p} 53$ binding sites in Nolc1 gene. (a) The positions of the putative p53 binding sites (BS from 1 to 4 ) in Nolc1 gene are represented as red bars on a USSC Genome Browser (Feb.2009 Assembly) depiction of the Nolc1 genomic sequence. (b) Sequence information about the putative p53 binding sites. The mismatches with the consensus p53 binding sequence ${ }^{1}$ and are highlighted in red. Shown are the distances (in base pairs) from the transcription start site (TSS). p53scan score is calculated with the following parameters: spacer length $s 0-1$, cutoff $c$
4.39 and 13.7 for $s=0$ and $s=1$ respectively, number of returned results $n$ 5 as described ${ }^{2}$. (c) Quantitative RT-PCR analysis of p21/WAF1 and Nolc1 mRNA in HCT116 WT cells after treatment with $5 \mathrm{mM}$ camptothecin or $0.5 \mathrm{mg} / \mathrm{ml}$ bleocin for 4 hours. The values are normalized to DMSO treated control, $t$-test $p$-values are denoted as * $-p<0.05$ and ** $-p<0,001$ (mean \pm s.d., $n=3$ ). (d) Response of Nolc1 and p21 promoter carrying luciferase reporter constructs to p53 WT overexpression in HCT116 WT and KO cells (mean \pm s.d., $n=3$ ). 
a

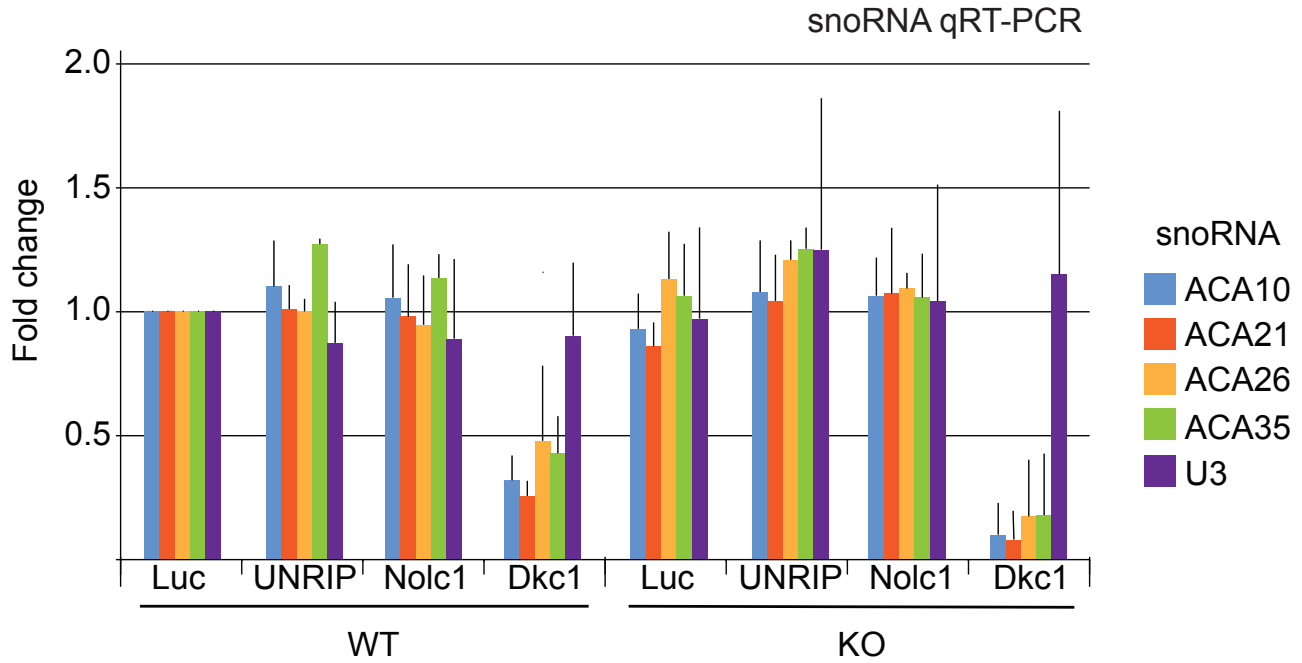

b

ACA10 (box H/ACA snoRNA)

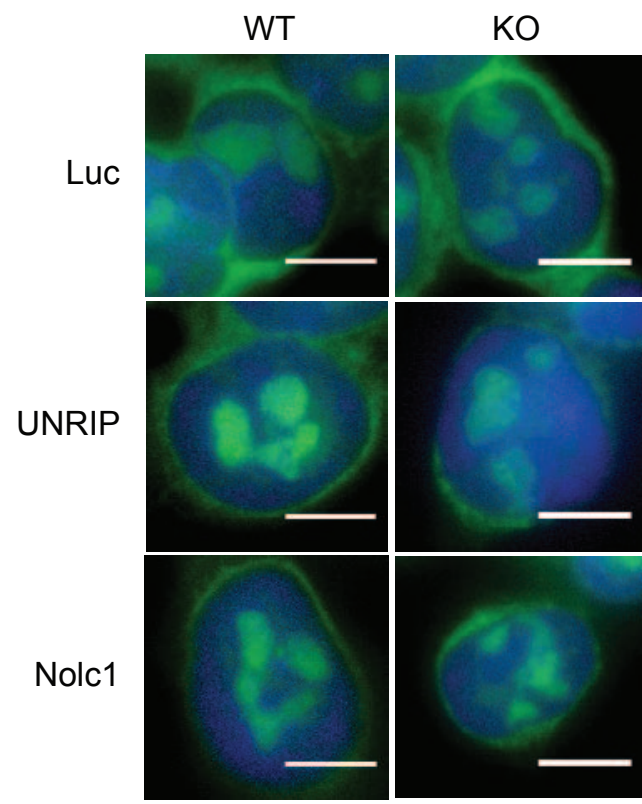

U3

(box C/D snoRNA)
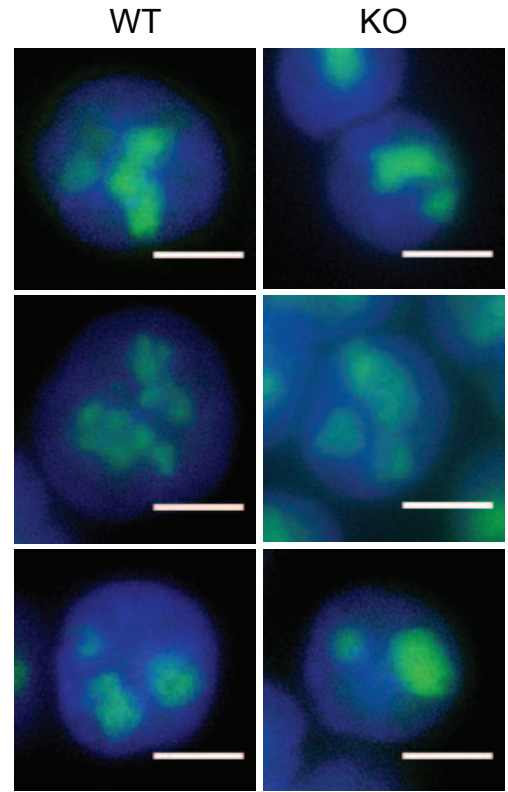

Figure S5 Nolc1 and UNRIP do not play a role in snoRNA stability and trafficking. (a) Quantification of the levels of five mature snoRNAs upon Nolc1, UNRIP or Dkc1 depletion in WT and KO cells. The values are normalized to
Luc depleted WT cells (mean $\pm s . d, n=3$ ). (b) RNA FISH shows the cellular localization of representative box H/ACA (ACA10) and box C/D (U3) snoRNAs upon UNRIP or Nolc1 depletion. Scale bar represents distance of $5 \mu \mathrm{m}$. 
a

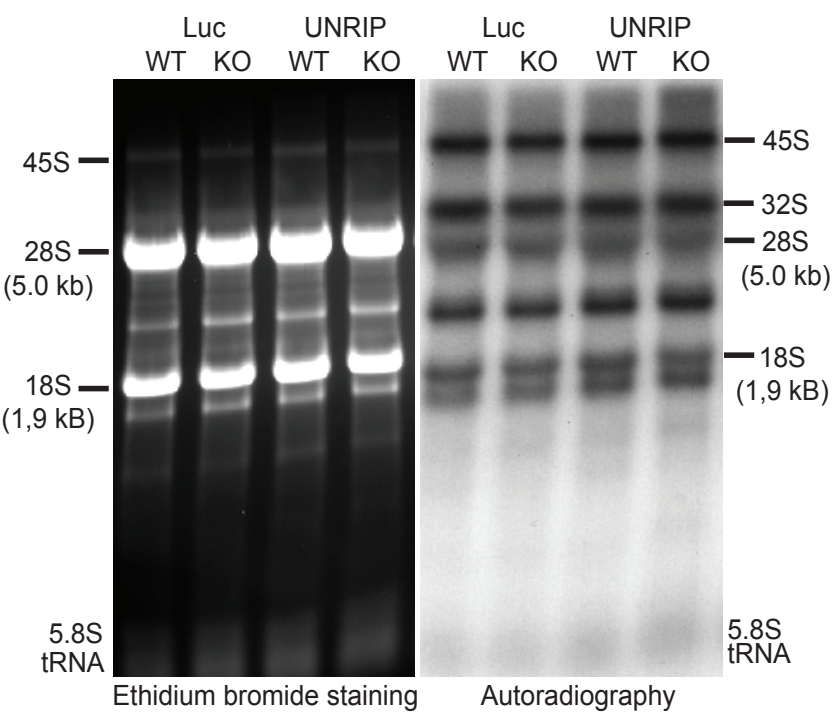

b

WT KO

WT KO Luc UNRIP Luc UNRIP

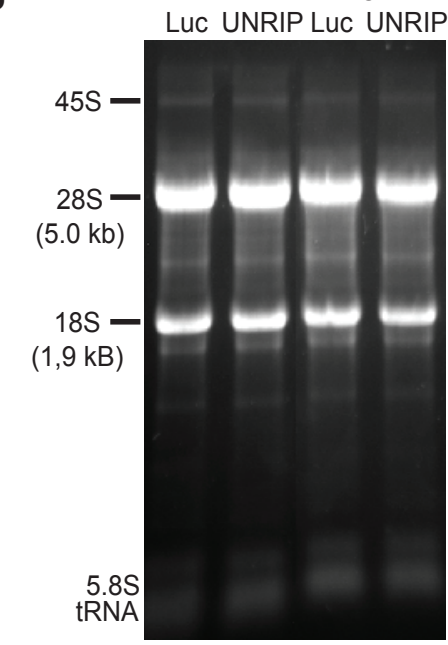

Ethidium bromide staining LUC UNRIP LUC UNRIP

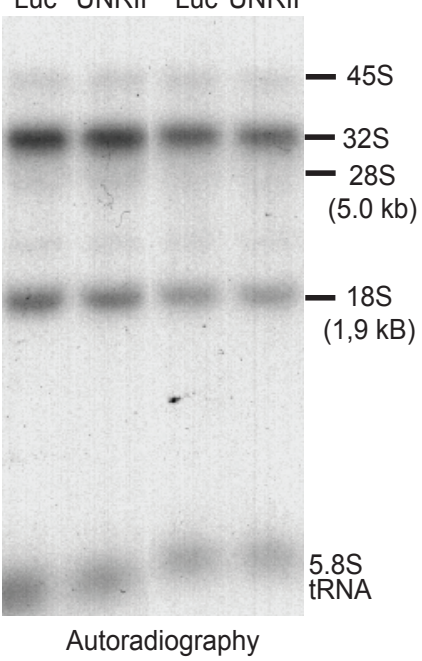

C

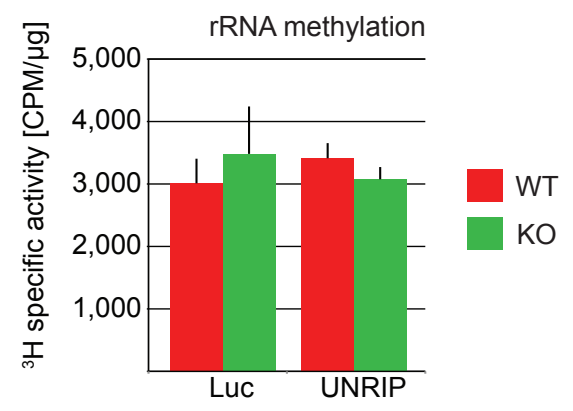

Figure S6 rRNA cleavage maturation and methylation are not affected by Nolc1 and UNRIP. (a) rRNA cleavage patterns were investigated in UNRIP depleted ${ }^{32} \mathrm{P}$-orthophosphate metabolically labeled cells. After $30 \mathrm{~min}$ chase period, $5 \mu \mathrm{g}$ total RNA were resolved on a denaturing agarose gel and autoradiographed. (b) rRNA methylation was investigated in UNRIP depleted L-[methyl-3H]-Methionine metabolically labeled cells. After 30 min chase period, $5 \mu \mathrm{g}$ total RNA were resolved on a denaturing agarose gel and autoradiographed. (c) Quantification of the incorporation of methyl groups in the rRNA, ${ }^{3} \mathrm{H}$ specific activity, measured on a scintillation counter. 
a

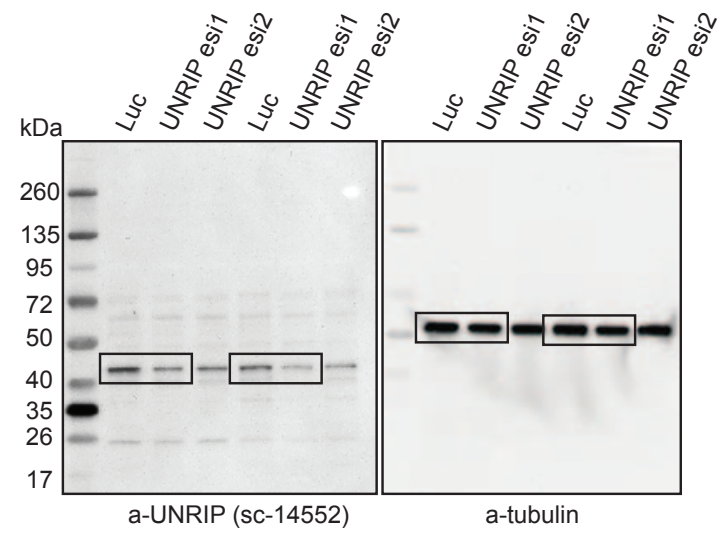

b
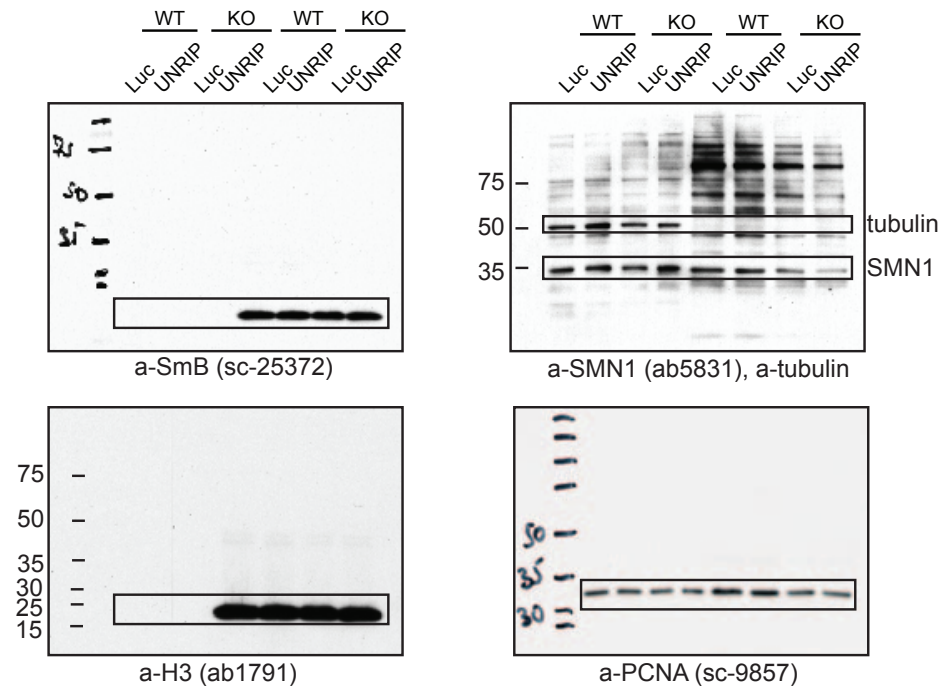

c
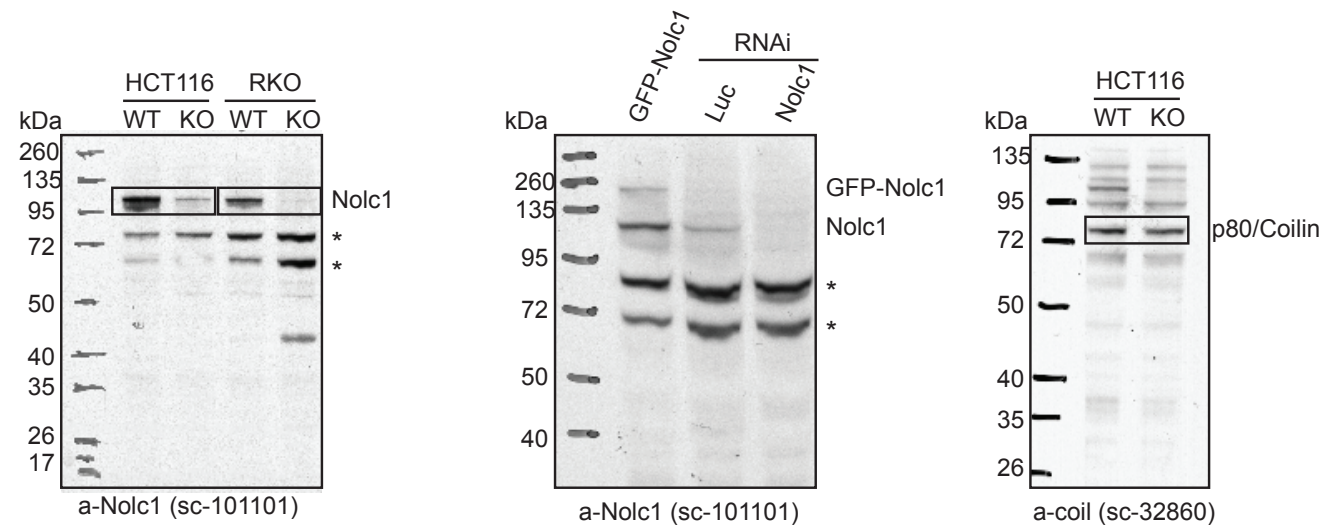

Figure S7 Full scans of blots. (a) Uncropped scans of UNRIP knockdown, Fig.2d and Fig. S2e. (b) Uncropped scans of the blots on Fig. 3c. (c)
Uncropped scans of the blots on Fig. 4c, * - unspecific bands (they cannot be depleted by RNAi and cannot be shifted by GFP-tagging). 
Table S1 Genome-scale screen primary data and calculated scores. A and B in the column names denote values obtained from the technical replicates of the screen.

Table S2 Validated screen hits with their scores and primers for esiRNA generation.

Table S3 Summary of MS analysis of UNRIP-LAP complexes. The 'Recovery' column summarizes how many times a protein was identified in total of 7 independent pulldown purifications.

Table S4 Primers used in the study.

\section{Supplementary References}

1. Riley, T., Sontag, E., Chen, P. \& Levine, A. Transcriptional control of human p53-regulated genes. Nat Rev Mol Cell Bio/ 9, 402-412 (2008).

2. Smeenk, L. et al. Characterization of genome-wide p53-binding sites upon stress response. Nucleic Acids Res, 17 (2008).

3. Mattia, M. et al. p53-dependent p21 mRNA elongation is impaired when DNA replication is stalled. Mol Cell Biol, 27 (2007). 